\title{
Inhibition of apolipoprotein B synthesis stimulates endoplasmic reticulum autophagy that prevents steatosis
}

\author{
Donna M. Conlon, Tiffany Thomas, Tatyana Fedotova, Antonio Hernandez-Ono, Gilbert Di Paolo, Robin B. Chan, \\ Kelly Ruggles, Sarah Gibeley, Jing Liu, and Henry N. Ginsberg
}

Department of Medicine, College of Physicians and Surgeons, Columbia University, New York, New York, USA.

\begin{abstract}
Inhibition of VLDL secretion reduces plasma levels of atherogenic apolipoprotein B (apoB) lipoproteins but can also cause hepatic steatosis. Approaches targeting apoB synthesis, which lies upstream of VLDL secretion, have potential to effectively reduce dyslipidemia but can also lead to hepatic accumulation of unsecreted triglycerides (TC). Here, we found that treating mice with apoB antisense oligonucleotides (ASOs) for 6 weeks decreased VLDL secretion and plasma cholesterol without causing steatosis. The absence of steatosis was linked to an increase in ER stress in the first 3 weeks of ASO treatment, followed by development of ER autophagy at the end of 6 weeks of treatment. The latter resulted in increased fatty acid (FA) oxidation that was inhibited by both chloroquine and 3-methyl adenine, consistent with trafficking of ER TC through the autophagic pathway before oxidation. These findings support the concept that inhibition of apoB synthesis traps lipids that have been transferred to the ER by microsomal TC transfer protein (MTP), inducing ER stress. ER stress then triggers ER autophagy and subsequent lysosomal lipolysis of TC, followed by mitochondrial oxidation of released FA, leading to prevention of steatosis. The identification of this pathway indicates that inhibition of VLDL secretion remains a viable target for therapies aiming to reduce circulating levels of atherogenic apoB lipoproteins.
\end{abstract}

\section{Introduction}

Dyslipidemia, the most common atherogenic lipid disorder, is characterized by increased production of VLDL, leading to high plasma levels of triglycerides (TG) and small, dense LDL and reduced concentrations of HDL cholesterol (1). Inhibition of the assembly and secretion of VLDL by the liver would have beneficial effects on all of these abnormalities but could worsen the hepatic steatosis that is commonly associated with dyslipidemia $(2,3)$. Secretion of VLDL requires both synthesis of apolipoprotein B (apoB) and microsomal TG transfer protein-mediated (MTP-mediated) delivery of surface and core lipids to nascent apoB during its cotranslational translocation across the ER (4). Both apoB and MTP are logical targets, therefore, for treatments to prevent cardiovascular disease by reducing the assembly and secretion of VLDL, the precursor of $\operatorname{LDL}(5,6)$. An antisense oligonucleotide (ASO) against apoB has been approved for the treatment of patients with homozygous familial hypercholesterolemia $(\mathrm{HoFH})$ and is effective at lowering plasma apoB and LDL-C levels (7). A small-molecule inhibitor of MTP has also been approved for patients with $\mathrm{HoFH}$ (8). Although it is unclear from early clinical trials whether one of these approaches will cause less steatosis than the other, we were intrigued by studies in rodents indicating that ASOmediated inhibition of apoB synthesis $(9,10)$ did not cause hepatic steatosis, whereas steatosis had been observed with both an ASO

Conflict of interest: H.N. Ginsberg has consulted for lonis Pharmaceuticals, which provided the antisense oligonucleotides for these studies. Submitted: December 14, 2015; Accepted: August 1, 2016.

Reference information: J Clin Invest. 2016;126(10):3852-3867. doi:10.1172/JCI86028. directed against MTP and small-molecule inhibitors of MTP activity (10-12). Here, we report that ASO-mediated inhibition of apoB synthesis, but not MTP, results in the accumulation of lipid in the ER, causing ER stress that, in turn, stimulates ER autophagy. The latter mediates lysosome-directed fatty acid (FA) oxidation of the ER lipids, thereby preventing steatosis. Our results indicate that inhibition of apoB synthesis or secretion should continue to be a target for the treatment of atherogenic dyslipidemia.

\section{Results}

In vivo hepatic $T G$ and $a p o B$ secretion was reduced to a similar extent by MTP and apoB ASO treatment. apobec-1-KO mice, which synthesize and secrete only apoB100, were placed on a high-fat diet (HFD) for 6 weeks and then injected for an additional 6 weeks with scrambled (control), MTP, or apoB ASOs ( $50 \mathrm{mg} / \mathrm{kg}$ biweekly i.p.). ASOs against either MTP or apoB selectively reduced mRNA levels of each by $80 \%$ and $97 \%$, respectively, after 6 weeks (Figure 1A). Knockdown of MTP and apoB was specific to the liver; small intestinal expression of Mttp and Apob was not significantly reduced after 6 weeks of ASO treatment (Supplemental Figure 1; supplemental material available online with this article; doi:10.1172/JCI86028DS1). There was no significant effect of either MTP or apoB ASO on BW (data not shown).

TG secretion was reduced similarly (70\%-85\%) with either MTP or apoB ASO compared with control ASO TG secretion (Figure 1B). The decreased secretion of TG was associated with $60 \%$ to $80 \%$ reductions in apoB100 secretion in the MTP ASO- and apoB ASO-treated groups, respectively (Figure $1 \mathrm{C}$ ). The similar reductions in TG and apoB secretion indicated that each treat- 

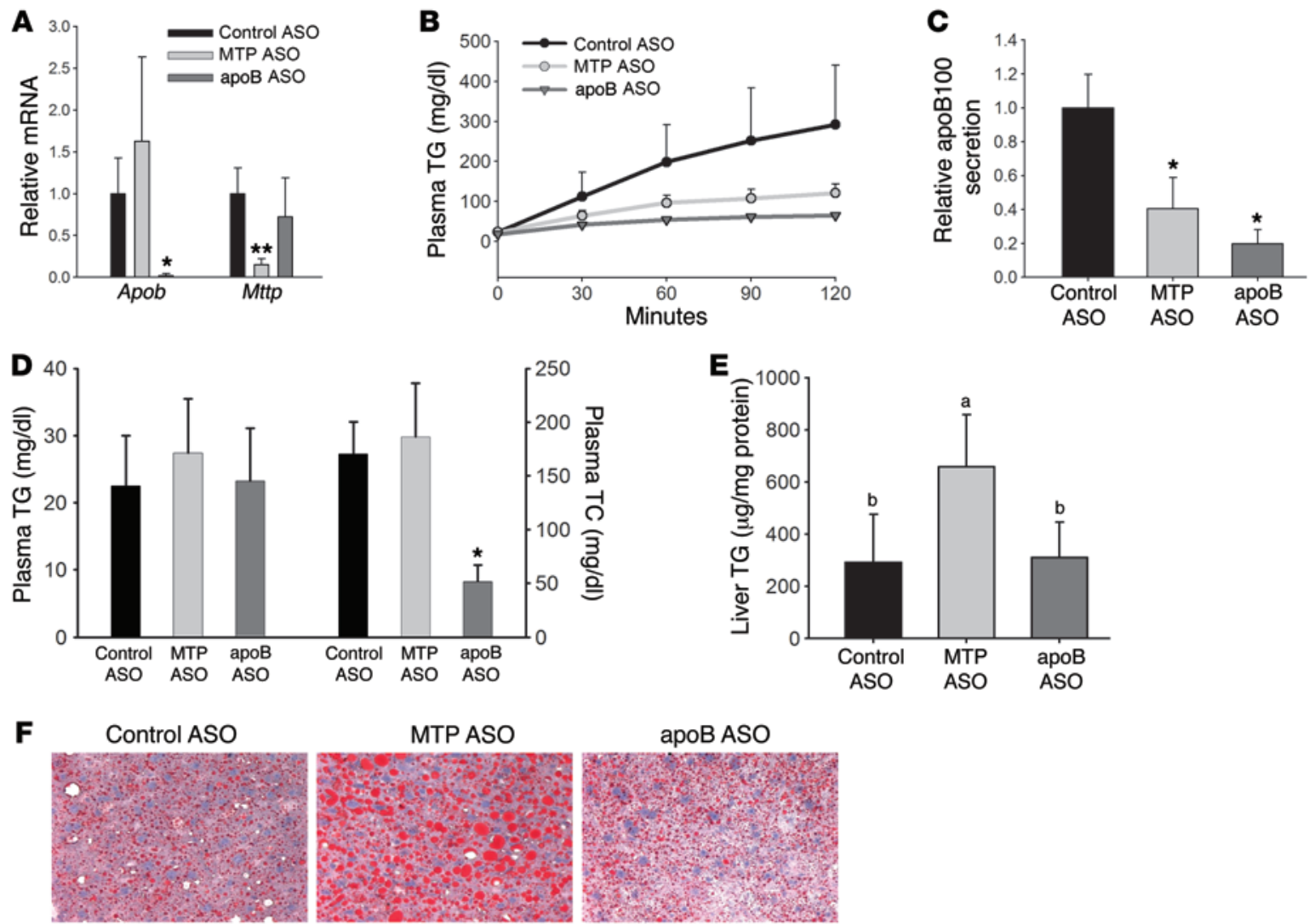

Figure 1. MTP ASO and apoB ASO reduce secretion of TG and newly synthesized apoB similarly, but only MTP ASO increases hepatic TG levels. (A) Measurement of Apob and Mttp mRNA in the liver of apobec-1-KO mice treated with ASO directed against MTP, apoB, or control for 6 weeks by real-time quantitative (qRT-PCR). Data were normalized to actin and are expressed relative to the ASO control group. $N=4-5$ per group. (B) apobec-1-KO mice were injected i.v. with Triton WR1339 and ${ }^{35} \mathrm{~S}$-methionine. Blood samples were obtained over the next 120 minutes. TC levels in plasma were measured enzymatically. $N=4-5$ per group. (C) apoB was isolated by 4\% SDS-PAGE from the 120-minute plasma samples in the Triton studies described in B, bands were cut, and radioactivity was quantitated by scintillation counting. Results are displayed as cpm in plasma relative to control ASO. The quantity of plasma loaded was adjusted for TCA-precipitable cpm. $N=5-6$ per group. ${ }^{*} P<0.05$ versus control ASO-treated group, by ANOVA. (D) TC and cholesterol levels were measured enzymatically in plasma from mice bled after a 4-hour fast. $N=12-15$ per group. TC, total cholesterol. (E) Liver TC was extracted from mice treated with control ASO, MTP ASO, or apoB ASO for 6 weeks. Liver TG levels were measured enzymatically and normalized to liver protein. $N=12-15$ per group. Groups with different letters above the bars are significantly different from the others at $P<0.05$, by ANOVA. (F) Representative images from $5-\mu \mathrm{m}$ liver sections that were stained for neutral lipid using oil red 0 . Original magnification, $\times 400$. All values represent the mean $\pm S D$. $(\mathbf{A}$ and $\mathbf{D}){ }^{*} P<0.05$ versus control ASO- and MTP ASO-treated groups, by ANOVA; ${ }^{* *} P<0.01$ versus control ASO- and apoB ASO-treated groups, by ANOVA.

ment resulted in the secretion of fewer, but similar-sized, VLDL particles as compared with the control. Plasma TG levels, which are very low in apobec-1-KO mice, were not altered by either apoB or MTP ASO treatment, but plasma cholesterol was significantly decreased with apoB ASO treatment (Figure 1D). This decrease was primarily in HDL cholesterol and present at both 3 and 6 weeks. Changes in HDL cholesterol levels were not associated with changes in either plasma apoA-I levels or hepatic levels of SR-B1 and ABCA1 (Supplemental Figures 2-5).

Worsening of hepatic steatosis occurred with 6-week MTP ASO treatment but not 6-week apoB ASO treatment. Despite similar decreases in the quantity of TG and the number of VLDL particles secreted during ASO treatments, knockdown of MTP for 6 weeks was associated with a 2.1-fold increase in liver TG concentrations compared with that detected in mice treated with control ASO, whereas knockdown of apoB was not associated with greater steatosis than that seen in controls (Figure 1E). As expected for mice consuming an HFD for 12 weeks, oil red O staining demonstrated steatosis in livers from all 3 groups. However, there were significantly larger and more numerous lipid droplets (LDs) in livers of MTP ASO-treated mice, whereas LDs in apoB ASO-treated livers did not differ from control ASO-treated livers (Figure 1F). Expression of PPAR $\gamma$ and related target genes for LD proteins, such as Fsp27 and Plin2 (also known as Adrp), were also increased in livers from MTP ASO- but not apoB ASO-treated livers (Supplemental Figure 6).

De novo hepatic lipogenesis, FA uptake, FA oxidation, and FA secretion were not different in mice treated with apoB ASO for 6 weeks. The absence of steatosis in mice treated with apoB ASO for 6 weeks, despite marked reductions in TG and apoB secretion, led us to look for compensatory changes in the other major pathways that maintain hepatic lipid homeostasis: de novo hepatic lipogenesis (DNL), FA uptake from plasma, and FA oxidation (2). Treatment with either MTP ASO or apoB ASO was associated with a general trend toward reduced expression of several lipogenic genes compared with the control ASO-treated group, but direct measurement of hepatic DNL by determining incorporation of 
A
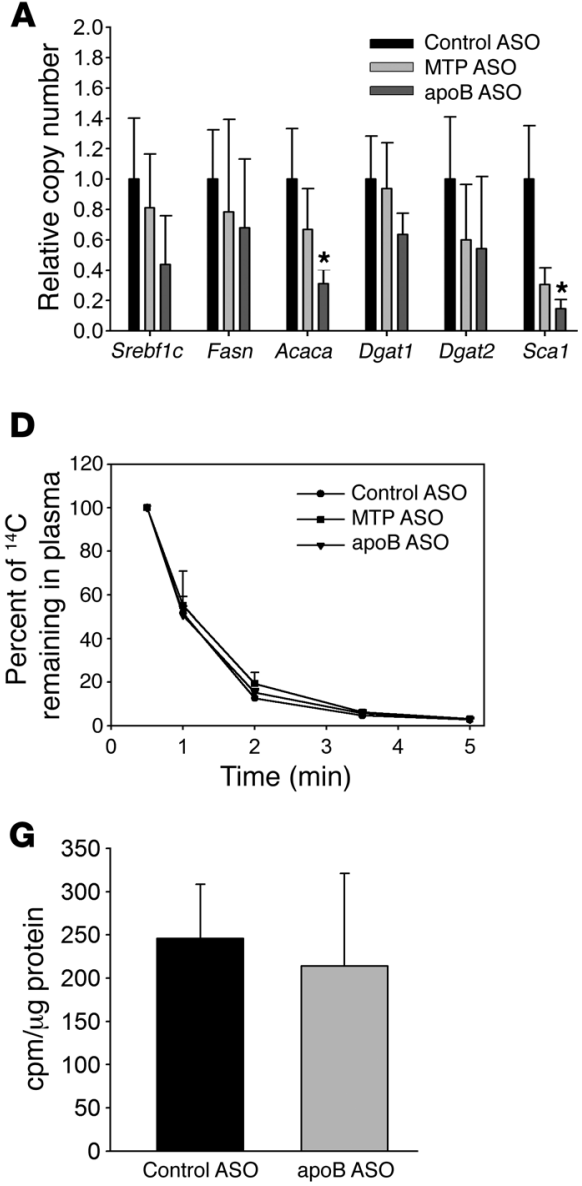

B

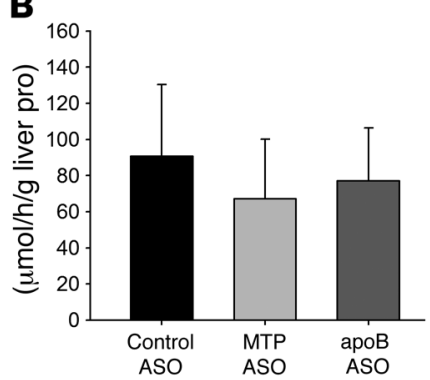

E

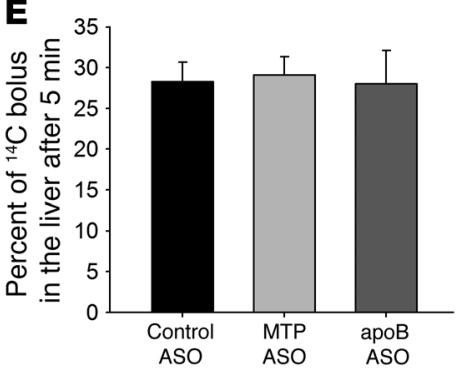

H

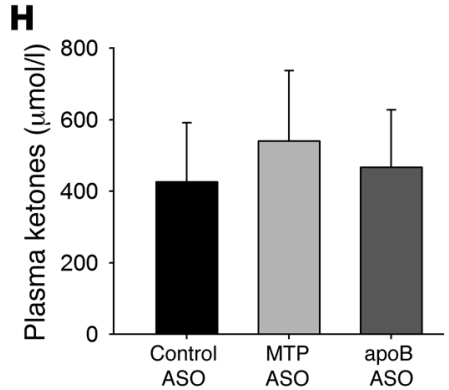

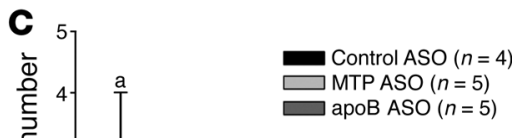

$\square \operatorname{MTP}$ ASO $(n=5)$
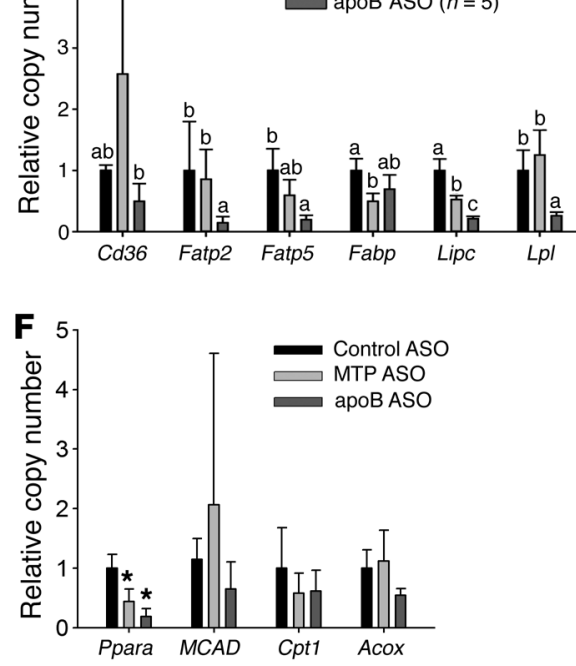

I

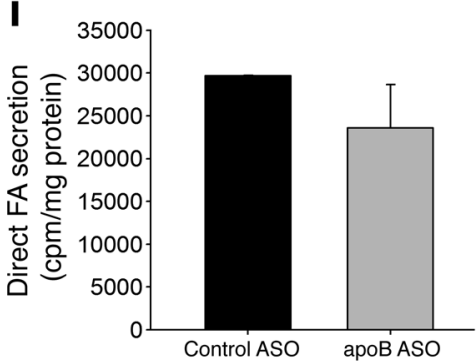

Figure 2. No consistent differences in hepatic DNL, FA oxidation, FA uptake, or FA secretion between apoB ASO- and MTP ASO-treated mice. (A) mRNA levels of genes related to lipid synthesis. $N=4-5$ per group. (B) Mice were injected i.p. with ${ }^{3} \mathrm{H}_{2} \mathrm{O}$, and the amount of newly synthesized liver lipids was measured after 1 hour. $N=6-8$ per group. (C) mRNA levels of genes related to hepatic uptake of plasma FA. $N=4$ per group. Groups with different letters above the bars are significantly different at $P<0.05$, by ANOVA. FATP2, fatty acid transport protein 2; FATP5, fatty acid transport protein 5; FABP, fatty acid binding protein; Lipc, hepatic lipase; Lpl, Lipoprotein lipase. (D) Mice were injected i.V. with ${ }^{14} \mathrm{C} O A$, and the disappearance of the labeled FA from plasma was measured over a 5-minute period. $N=3-6$ per group. (E) The amount of labeled FA uptake by the liver was measured after 5 minutes in the same mice. $N=3-6$ per group. (F) mRNA levels of genes related to FA oxidation. $N=4-5$ per group. PPARA, peroxisome proliferator activated receptor $\alpha$; $M C A D$, Medium-chain acyl-CoA dehydrogenase; Cpt1, Carnitine palmitoyltransferase 1; Acox, acyl-coenzyme A oxidase. (C) Primary hepatocytes from control ASOand apoB ASO-treated mice were labeled for 2 hours with ${ }^{14} \mathrm{C} O \mathrm{OA}$, and then the amount of ${ }^{14} \mathrm{CO}_{2}$ and ${ }^{14} \mathrm{C}$ ASMs released into the media was measured and normalized to cell protein levels. $N=12$ wells from 4 mice per group. (H) An enzymatic assay was used to measure total plasma ketones in plasma taken from mice treated for 6 weeks with ASO. $N=6-10$ per group. (I) Primary hepatocytes from control ASO- and apoB ASO-treated mice were labeled with ${ }^{14} \mathrm{C}$ OA for 16 hours and then chased with unlabeled media for 4 hours. Lipid was extracted from the chase media and separated by TLC. The amount of ${ }^{14} \mathrm{C}$ FA was counted and normalized to total cell protein as a measure of direct FA secretion. $N=9$ wells from 3 mice per group. All values represent the mean \pm SD. $(\mathbf{A}$ and $\mathbf{F}){ }^{*} P<0.05$ versus control ASO-treated mice, by ANOVA.

${ }^{3} \mathrm{H}_{2} \mathrm{O}$ into hepatic FAs showed no significant difference across the 3 treatment groups after 6 weeks of ASO treatment (Figure 2, A and $\mathrm{B})$. Congruent with this observation, Lee et al. reported reductions in the expression of lipogenic genes after several weeks of treatment of $\mathrm{C} 57 \mathrm{Bl} / 6 \mathrm{~J}$ mice with the same apoB ASO that we used, but when DNL was measured biochemically in vivo, those investigators observed no significant difference between apoB ASO- and control ASO-treated mice (10).

We observed significant decreases in the expression of several genes associated with FA uptake from plasma in apoB ASO-treated mice, but there were no differences in the in vivo uptake of plasma FAs among the 3 groups, as indicated by both the similar rates of FA clearance from plasma and the hepatic uptake of i.v.-injected
BSA-bound ${ }^{14} \mathrm{C}$ oleic acid (OA) (Figure 2, C-E). Knockdown of either MTP or apoB was not associated with significant changes in the expression of genes related to lipid oxidation (Figure 2F). We assessed FA oxidation directly in cultured primary hepatocytes isolated from control ASO- and apoB ASO-treated mice (we were unable to isolate viable primary hepatocytes from MTP ASOtreated mice due to the extreme degree of steatosis in those cells). There was no significant difference in the amount of ${ }^{14} \mathrm{CO}_{2}$ plus ${ }^{14} \mathrm{C}$ acid-soluble metabolites (ASMs) produced during 2 hours of labeling with ${ }^{14} \mathrm{C}$ OA between primary hepatocytes isolated from apoB ASO- and control ASO-treated mice (Figure 2G). There was also no difference in total plasma ketone concentrations between the control ASO-, MTP ASO-, and apoB ASO-treated mice (Figure 
A Control ASO
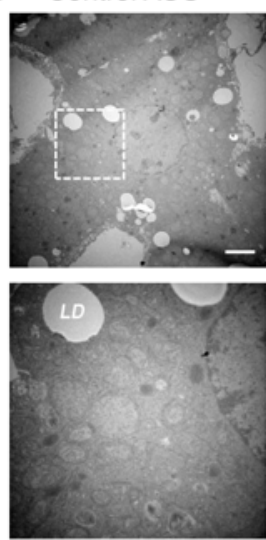

C

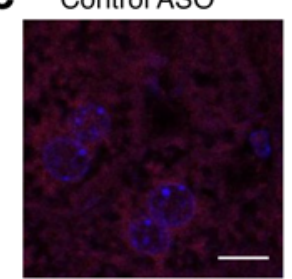

MTP ASO
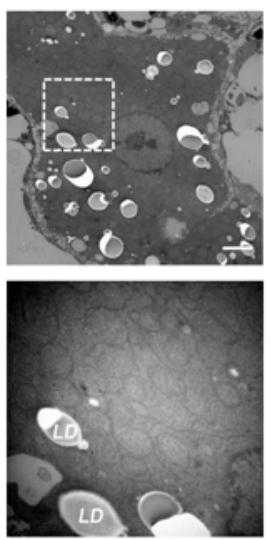

MTP ASO

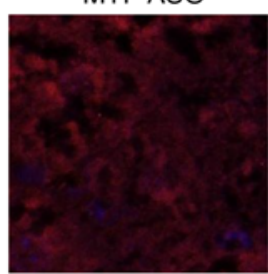

apoB ASO
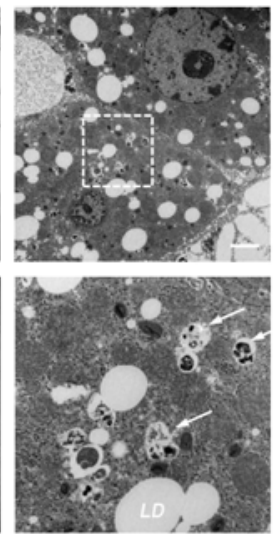

apoB ASO

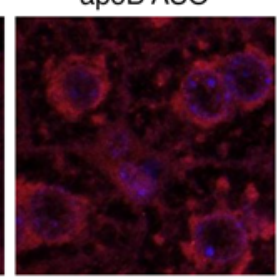

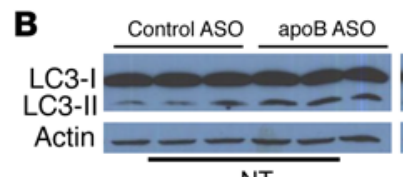

NT
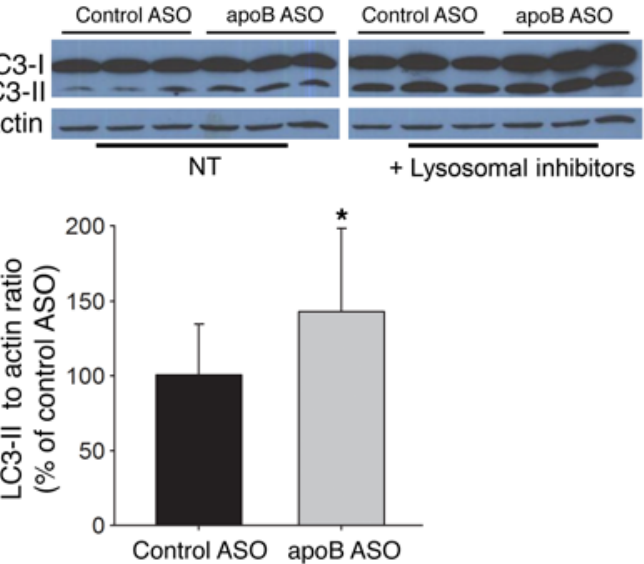

D

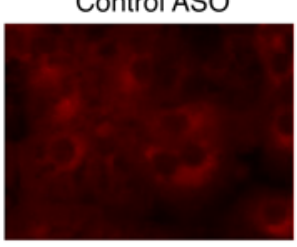

apoB ASO

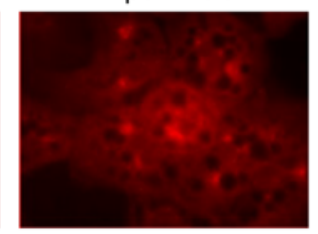

Figure 3. apoB ASO-treated livers have an increase in autophagosome structures as well as increased LC3-II levels. (A) Representative EM images (original magnification, $\times 6,000$ and $\times 20,000$ [magnified insets, bottom row]) of livers from control ASO-, MTP ASO-, and apoB ASO-treated mice. N = 3 livers per group. Scale bars: $2 \mu \mathrm{m}$. (B) Immunoblots of LC3-I and LC3-II (separated by SDS-PACE); the PE-linked LC3-II runs faster on the gel. Left gel, with normal lysosomal activity, was exposed for 1 minute; right gel, with inhibition of lysosomes, was exposed for 1 second, because with lysosomal inhibition, both LC3-I and LC3-II were significantly increased. Immunoblots of the nontreated (NT) lysates for LC3-II bands were quantified using ImageJ (NIH) densitometry. $N=12$ per group. Values represent the mean $\pm \mathrm{SD}$. ${ }^{*} P<0.05$ between groups, by Student's $t$ test. (C) Livers from apobec-1-KO mice treated with each ASO for 6 weeks were incubated with anti-LC3 Ab (red) and stained with DAPI (blue). Images were taken using a NikonA1RMP confocal microscope (original magnification, $\times 600$ ). Depicted are representative images from 3 livers per group, 5 images per liver. Scale bar: $20 \mu \mathrm{m}$. (D) Primary hepatocytes isolated from control ASO- and apoB ASO-treated mice were incubated with anti-LC3 Ab (red). Images were visualized using a Zeiss Axiovert 200M microscope (original magnification, $\times 200$ ). Depicted are representative images from 2 hepatocyte isolations per group. $N=5$ images per isolation.

2H). Another possible pathway for the decrease in lipid accumulation in the apoB ASO-treated livers is an increase in the direct hepatic secretion of FAs (13). However, there was no difference in the amount of ${ }^{14} \mathrm{C}$-labeled FAs secreted from control ASO- and apoB ASO-treated hepatocytes (Figure 2I).

apoB ASO treatment for 6 weeks is associated with a marked increase in autophagy. Because we could not explain the lack of hepatic lipid accumulation in the apoB ASO-treated livers, electron microscopy (EM) was performed on liver samples from mice treated with each ASO. Livers from mice treated for 6 weeks with apoB ASO had a marked increase in autophagosome-like organelles throughout the cell (Figure 3A). To investigate this finding further, we measured levels of the autophagosomal marker microtubule-associated protein 1A/1B-light chain 3 (LC3-II) by immunoblotting in primary hepatocytes isolated from control ASO- and apoB ASO-treated mice. LC3-II is the lipidated form of LC3 that is required for incorporation of this protein into the membrane of the autophagosome and is a marker of autophagy from formation of the phagophore until fusion with the lysosome. There was a significant increase in the amount of LC3-II in the apoB ASO-treated hepatocytes compared with the amounts detected in control ASO-treated cells. Treatment of the hepatocytes with lysosomal inhibitors showed accumulation of LC3-II protein in both groups, with greater accumulation in the apoB ASO-treated hepatocytes, indicating that the increase in LC3-II protein in apoB ASO-treated mice was due to increased autophagosome formation and not to a defect in autophagic flux (14) (Figure 3B).

We next performed immunofluorescence staining on $5-\mu \mathrm{m}$ liver sections from control ASO-, apoB ASO-, and MTP ASOtreated mice: total LC3 staining was increased only in the apoB ASO-treated livers (Figure $3 \mathrm{C}$ ). In contrast, MTP ASO-treated livers, despite the presence of marked steatosis, did not have increased LC3 staining; this may have been due to the HFD the mice were consuming, which can inhibit autophagy (15). Increased LC3 staining in apoB ASO-treated liver sections was perinuclear, indicative of the presence of more mature autolysosomes (Figure 3C) (16). There was a marked increase in LC3 staining in primary hepatocytes isolated from apoB ASO-treated mice as compared with that seen in primary hepatocytes from control ASO-treated mice (Figure 3D). There were also increased lysosomes, as seen by immunofluorescence staining for lysosomal-associated membrane protein 2 (LAMP2), which is further evidence of an increase in hepatic autophagy (Supplemental Figure 7A).

P62 (also known as SQSTM1) is used as a marker of defective autophagy (17). We examined levels of liver p62 in all 3 groups of mice at 3 and 6 weeks and did not observe differences by group at either time point (Supplemental Figure 8), supporting our conclusion that increased LC3-II levels are indicative of increased 
A

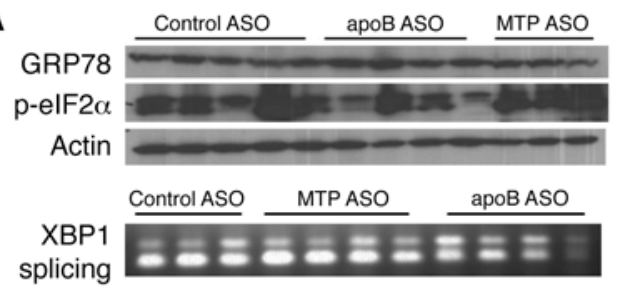

B

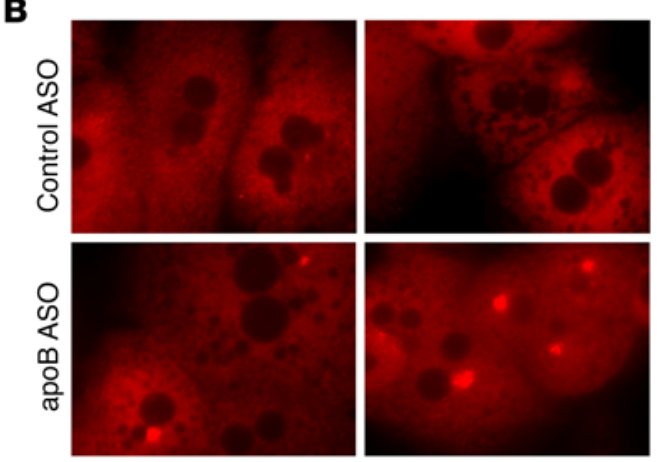

C

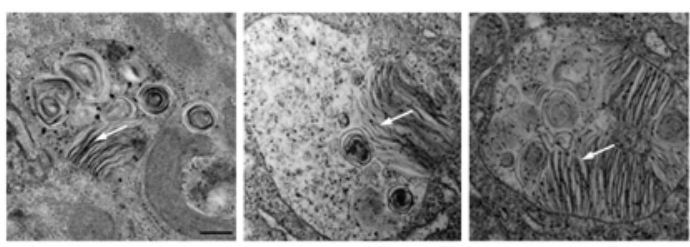

D
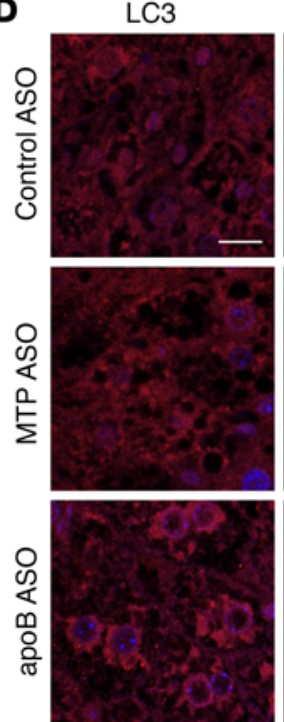

LC3
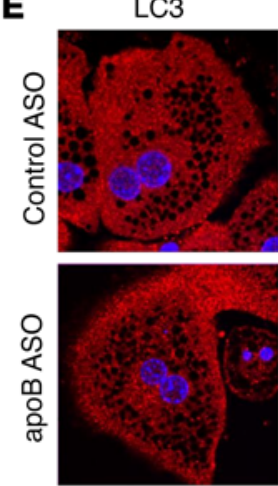

Calnexin
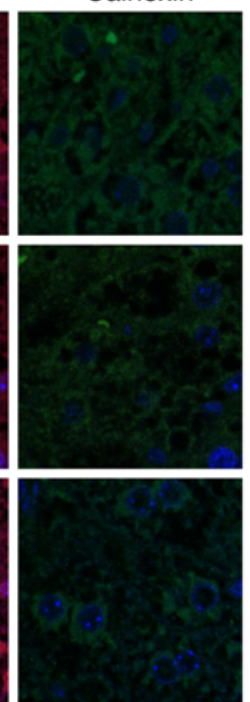

Calnexin
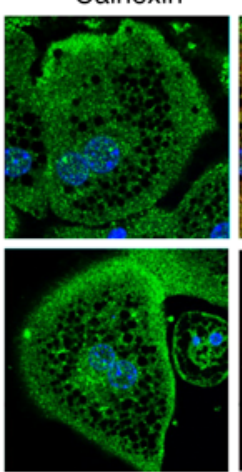

Merge
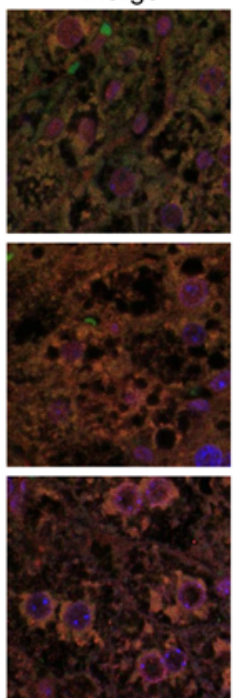

Merge
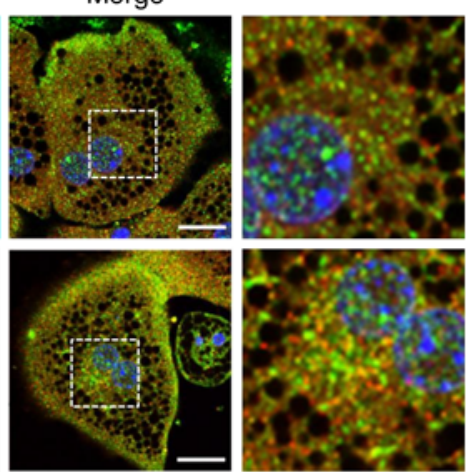

Figure 4. apoB ASO-treated livers have evidence of increased autophagy of ER, with no change in ER stress levels. (A) Liver homogenates from control ASO-, apoB ASO-, and MTP ASO-treated mice were separated by SDS-PAGE and immunoblotted for phoso-elF2 $\alpha$, GRP78, and actin. Representative immunoblots are shown. CDNA from the same ASO-treated livers was digested by Pstl. The upper band represents the spliced form of X-box-binding protein 1 (XBP1). $N=8-12$ per group. (B) Primary hepatocytes were stained with ER-Tracker Red for 30 minutes. Images were taken with a Zeiss Axiovert $200 \mathrm{M}$ microscope (original magnification, $\times 200$ ). $N=2$ hepatocyte isolations per group; $N=5$ images per group. (C) Representative autolysosomes from apoB ASO-treated livers (see Figure 1G) (original magnification, $\times 20,000$ ) showing lamellae-like structures. Scale bar: $100 \mathrm{~nm}$. (D) Representative images of liver sections that were incubated with anti-LC3 Ab (red) and anti-calnexin Ab (green) and then stained with DAPI (blue). Images were taken with a Nikon A1RPM microscope (original magnification, $\times 600$ ). $N=3$ livers per group; 5 images per liver. Scale bar: $20 \mu \mathrm{m}$. (E) Primary hepatocytes isolated from control ASO- and apoB ASO-treated mice were incubated with anti-LC3 Ab (red) and anti-calnexin Ab (green) and then stained for DAPI (blue). Images were visualized using a Nikon Ti Eclipse inverted confocal microscope and a $60 \times / 1.49$ NA oil lens. $N=3$ hepatocyte isolations per group; 3 images per isolation. Scale bars: $10 \mu \mathrm{m}$.

autophagic flux rather than of defects in the pathway. Decreased levels of p62 are not considered to be a consistent marker of increased autophagy $(18,19)$.

apoB ASO treatment for 6 weeks causes abnormalities in the ER. Although apoB synthesis is markedly reduced in the livers of apoB ASO-treated mice, MTP is still present and able to transfer lipid from the ER membrane into the lumen of the ER where, with the near absence of apoB available to secrete the lipid, we believe it would accumulate. Since lipids have been shown to induce ER stress $(20,21)$, we examined several markers of ER stress and the unfolded protein response (UPR) in apoB ASO-treated livers. Surprisingly, there was no increase in phosphorylated eIF $2 \alpha$ (p-eIF $2 \alpha$ ), total GRP78, or spliced XBP1 in the livers of mice treated for 6 weeks with either apoB ASO or MTP ASO (Figure 4A; den- sitometry in Supplemental Figure 9). However, when we stained primary hepatocytes with ER-Tracker Red, we observed abnormal aggregates of ER in apoB ASO-treated cells (Figure 4B). Furthermore, examination of the autophagosomes identified in the electron micrographs revealed the presence of lamellar structures resembling ER (Figure 4C) (22). Because the absence of steatosis in the apoB ASO-treated mice had become our focus and, as well, we were unable to isolate primary hepatocytes from MTP ASOtreated mice, all further studies on primary hepatocytes compared only control ASO- and apoB ASO-treated mice.

apoB ASO treatment for 6 weeks is associated with increased autophagy of the ER. Given these results, we hypothesized that there was an increase in ER autophagy. We next performed immunofluorescence staining for LC3 anad calnexin, the latter as a mark- 
A

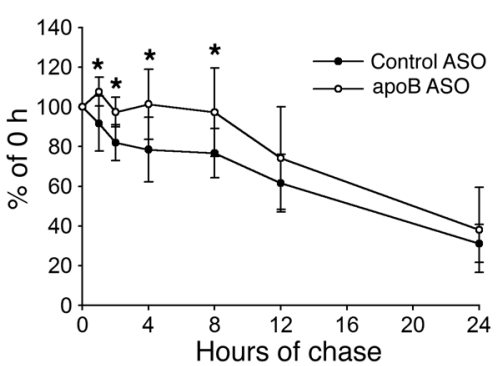

C

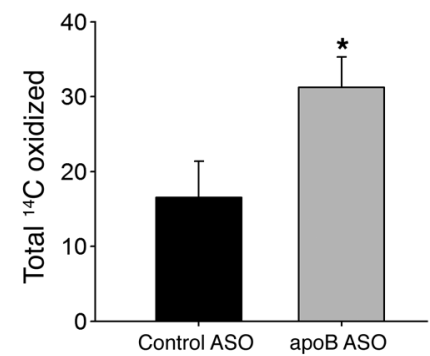

E

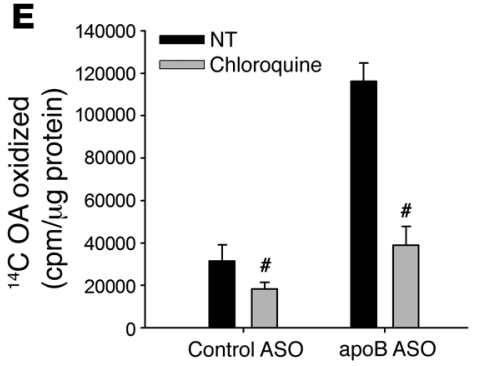

G

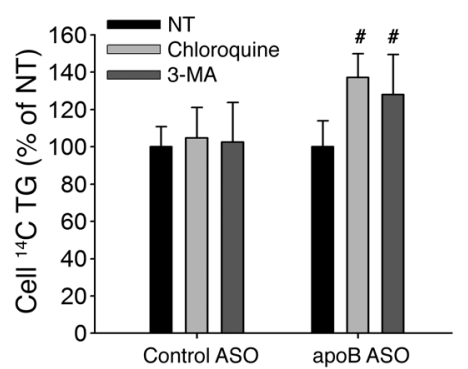

B

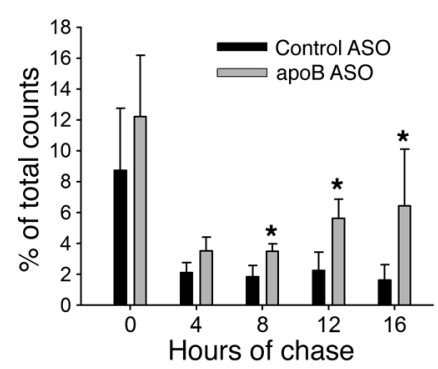

D

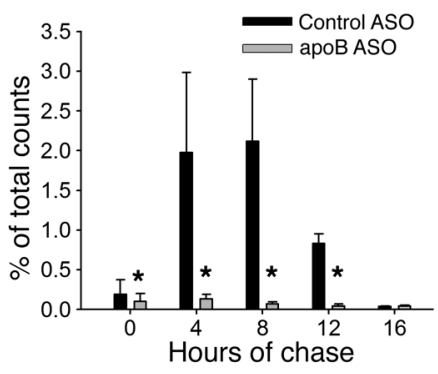

$\mathbf{F}$

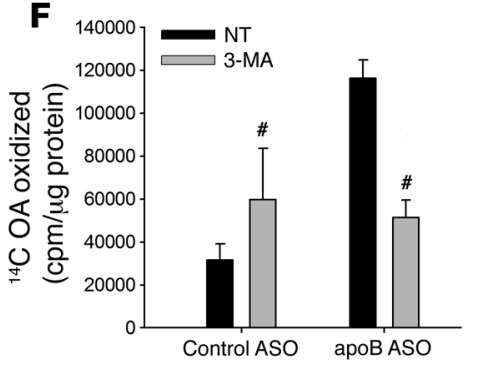

H

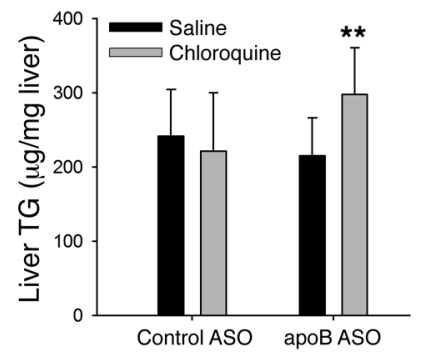

Figure 5. Time-course FA oxidation shows a delayed increase in oxidation of labeled FA in the apoB ASOtreated hepatocytes that is inhibited by blocking either lysosomal activity or autophagy. (A) Primary hepatocytes were labeled with ${ }^{3} \mathrm{H}$ glycerol for 2 hours and chased for either $0,1,2,4,8,12$, or 24 hours, after which cellular lipid was extracted and ${ }^{3} \mathrm{H}$ TC levels measured. Results are a percentage of total ${ }^{3} \mathrm{H}$ TC at 0 hours. $N=9-18$ per time point. (B) Hepatocytes were labeled with ${ }^{14} \mathrm{C} O A$ for 2 hours, and then unlabeled chase media were replenished every 4 hours over a 16-hour period. The amount of ${ }^{14} \mathrm{CO}_{2}$ and ${ }^{14} \mathrm{C}$ ASM is presented as a percentage of total ${ }^{14} \mathrm{C}$ counts at $\mathrm{O}$ hours for each 4-hour chase period. $N=5$ mice per group. (C) Total amount of ${ }^{14} \mathrm{COA}$ oxidized over the entire 16 -hour incubation period. (D) Media from parallel plates were collected, and the amount of ${ }^{14} \mathrm{C}$ TC secreted from the hepatocytes is represented as the percentage of total counts in the cell at 0 hours. $N=3$ wells per group. (E and F) Primary hepatocytes were labeled with ${ }^{14} \mathrm{C} \mathrm{OA}$ for 16 hours and then chased for 4 hours in the presence or absence of (E) $50 \mu \mathrm{M}$ chloroquine or (F) $5 \mathrm{mM} 3-\mathrm{MA}$, after which ${ }^{14} \mathrm{CO}_{2}$ and ${ }^{14} \mathrm{C}$ ASMs were measured. (C) After the end of the chase, cellular lipid was extracted, and ${ }^{14} \mathrm{C}$ TC was measured and normalized to no treatment. $N=6-9$ wells from 2 to 3 mice per group. (H) Mice were injected with ASO for 6 weeks and then injected with either saline or $60 \mathrm{mg} / \mathrm{kg}$ chloroquine for 10 days. Liver TC levels were measured and normalized to liver protein levels. $N=5-7$ per group. All values represent the mean \pm SD. (A-D) ${ }^{*} P<0.05$, by ANOVA, for apoB ASO-treated versus control ASO-treated hepatocytes. (E-G) ${ }^{\#} P<0.05$ versus no treatment, by ANOVA. $(\mathrm{H})$ ${ }^{*} P<0.05$, by ANOVA, for apoB ASO plus chloroquine versus apoB ASO plus saline. er of ER, on liver sections from control ASO-, MTP ASO-, and apoB ASO-treated mice (Figure 4D). In addition to the previously observed increase in LC3 staining, we found an increase in total calnexin staining, indicating the presence of more ER in the apoB ASO-treated group. When the 2 stains were merged, we observed clear colocalization of LC3 and calnexin, indicative of an increase in autophagy of the ER. This colocalization was not seen when immunofluorescence staining was performed for LC3 and voltage-dependent anion channel 3 (VDAC3), a marker of mitochondria, or perilipin 2 (PLIN2), a marker of LDs (Supplemental Figure 7B). There was, however, colocalization of LC3 with LAMP2, a lysosomal membrane marker, in the apoB ASO-treated livers, especially around the nucleus (Supplemental Figure 7B). We confirmed these results in primary hepatocytes costained for LC3 and calnexin (Figure 4E). In addition, we did not see an increase in PLIN2 or LC3 colocalization in isolated primary hepatocytes (Supplemental Figure 7C). The specificity of the observed autophagy for retained ER lipids was supported by the absence of any effect of apoB ASO treatment on the secretion of other proteins, such as albumin or apoA-I, from the liver (Supplemental Figure 4).

apoB ASO treatment for 6 weeks results in decreased TG turnover as a result of compartmentalization of lipid. Our results to this point provided strong support for the stimulation of ER autophagy in response to the inability to secrete VLDL. However, the increased transfer of lipids trapped in the lumen of the ER to the autophagic pathway would not, by itself, account for the absence of increased steatosis in livers of apoB ASO-treated mice compared with livers from control ASO-treated mice. We hypothesized that if there was an increase in autophagy of the ER, as was observed in apoB ASO-treated livers, any TG that had entered the ER lumen would become "trapped" in the ER and eventually become cargo within the autophagic pathway. Such compartmentalization of TG in ER and autophagosomes would result in a decrease in the rate of turnover of TG in the apoB ASO-treated livers. To determine whether 
A

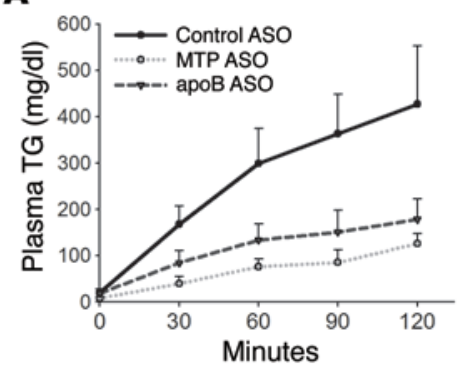

B

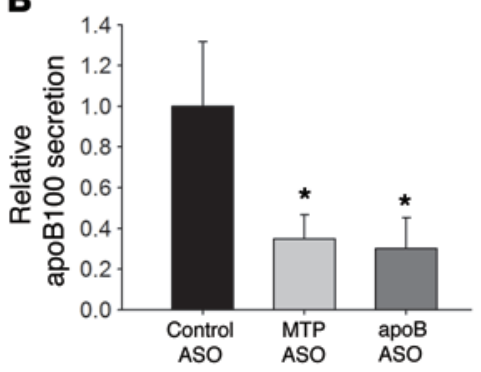

C

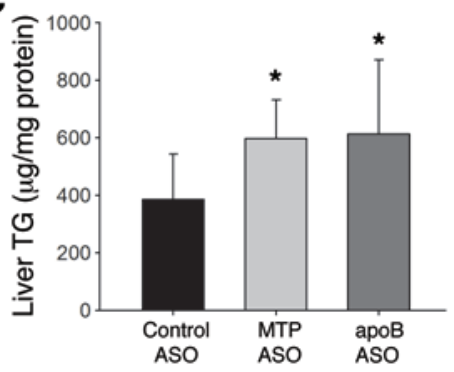

D

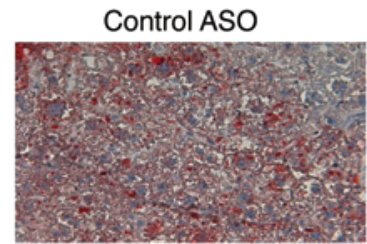

E

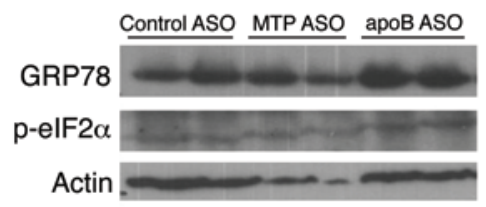

MTP ASO

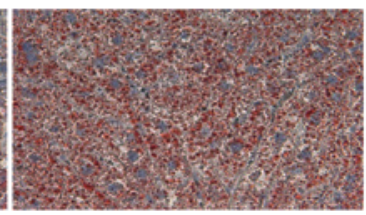

apoB ASO

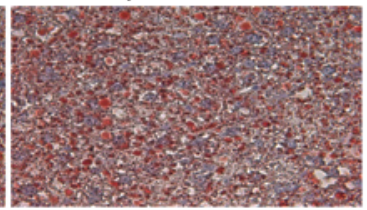

$\mathbf{F}$

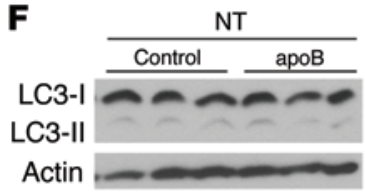
Lysomal inhibitor

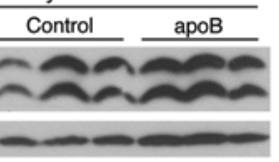

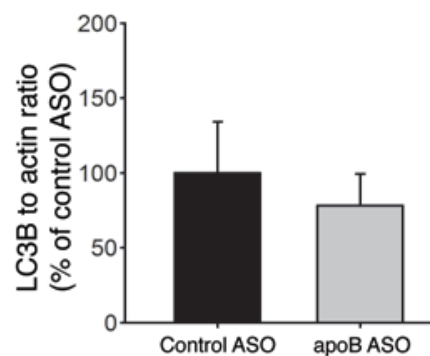

G

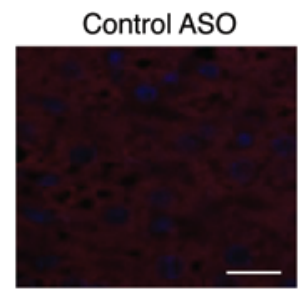

MTP ASO

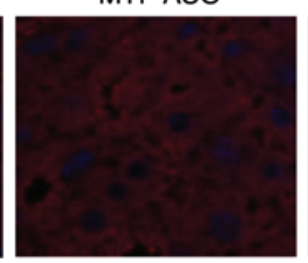

apoB ASO

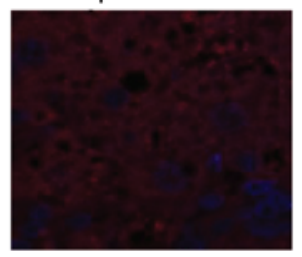

H

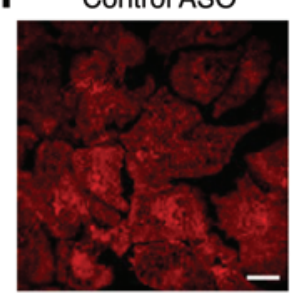

apoB ASO

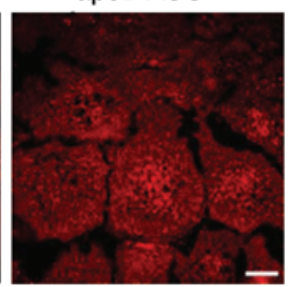

Figure 6. Treatment with apoB ASO for 3 weeks results in increased steatosis and increased ER stress but no change in autophagy. Triton WR1339 and ${ }^{35} \mathrm{~S}-$ methionine were injected i.v. into apobec-1-KO mice that were treated for 3 weeks with control, MTP, or apoB ASO, and blood samples were obtained over the next 120 minutes. (A) TG levels in plasma were measured enzymatically. (B) apoB was isolated by $4 \%$ SDS-PACE of the 120 -minute plasma sample, and bands were cut and counted. $N=5$ mice per group. (C) Liver lipid was extracted from mice treated with control ASO, MTP ASO, or apoB ASO for 3 weeks. Liver TG was measured enzymatically and normalized to liver protein. $N=13-14$ per group. (B and C) ${ }^{*} P<0.05$ for both MTP ASO- and apoB ASO- versus control ASO-treated mice, by ANOVA. Values represent the mean \pm SD. (D) Liver sections (5- $\mu$ m) were stained for neutral lipid using oil red 0 . Representative images are shown (original magnification, $\times 400$ ). $N=4$ livers per group with 5 images per liver. (E) Liver homogenate was separated by SDS-PAGE and immunoblotted for p-elF2 $\alpha$, GRP78, and actin. Representative blots are shown. $N=6$ livers per group. (F) Representative immunoblots of LC3-I and LC3-II with or without lysosomal inhibition. Graph shows quantification by Image) densitometry of immunoblots of the nontreated LC3-II bands. $N=6$ per group. (G) Livers from apobec-1-KO mice treated with each ASO for 3 weeks were incubated with anti-LC3 Ab (red) and then stained with DAPI (blue). Images were taken with a NikonA1RMP confocal microscope (original magnification, $\times 600$ ). $N=3$ mice per group; 5 images per mouse. Scale bar: $20 \mu \mathrm{m}$. (H) Primary hepatocytes isolated from mice treated for 3 weeks with either control ASO or apoB ASO were incubated with anti-LC3 Ab (red) and stained with DAPI (blue). $N=3$ hepatocyte isolations per group; 3 images per isolation. Scale bars: $10 \mu \mathrm{m}$.

there was a slowed TG turnover in the apoB ASO-treated hepatocytes, we labeled primary hepatocytes with ${ }^{3} \mathrm{H}$ glycerol for 2 hours in order to create a pool of ${ }^{3} \mathrm{H}$-labeled TG. We then measured how much of the labeled TG remained in the cell over a 24-hour period (Figure 5A). Over the first 8 hours, almost all of the ${ }^{3} \mathrm{H}$-labeled TG remained in the apoB ASO-treated hepatocytes, while a steady decline in these labeled TG in the control ASO hepatocytes was observed over the same time period. However, between 8 hours and 24 hours, the amount of ${ }^{3} \mathrm{H}$ TG remaining in the apoB ASO- treated liver cells began to decline. At 24 hours, there was no difference in the amount of ${ }^{3} \mathrm{H}$-labeled TG remaining in hepatocytes of the 2 groups. It is important to note that the delayed disappearance of TG occurred in the absence of any significant secretion of ${ }^{3} \mathrm{H}$-labeled TG by the apoB ASO-treated hepatocytes over the entire time course of the experiment. Additionally, this slowed turnover was unique to TG, as there was no difference in the disappearance of phospholipid (PL) in the hepatocytes from control APO- and apoB ASO-treated mice (data not shown). 
There is delayed but increased FA oxidation in 6-week apoB ASO-treated livers. Lipolysis of lipids within autolysosomes, with delivery of their FAs to mitochondria, could lead to oxidation that would compensate for the lack of secretion of VLDL, but our studies of FA oxidation in primary hepatocytes had not demonstrated differences between cells from control ASO- and apoB ASO-treated mice (Figure 2G). We considered the possibility that the 2-hour labeling-collection protocol used for those studies was too short to observe the oxidation of ${ }^{14} \mathrm{C}$ OA which, after incorporation into lipids in the ER membrane, would have to move to the lumen, then be incorporated into autophagosomes that would have to fuse to lysosomes before the lipids could be hydrolyzed to allow released ${ }^{14} \mathrm{C}$ OA to finally be oxidized by mitochondria. This schema is supported by published data demonstrating that the peak fusion of autophagosomes with lysosomes can occur as late as 16 hours after stimulation of autophagy $(23,24)$.

To test this model of delayed oxidation, we performed a timecourse FA oxidation study, in which we once again labeled primary hepatocytes with ${ }^{14} \mathrm{C}$ OA for 2 hours, but then chased the cells for 16 hours with label-free media, changing the media every 4 hours. At the end of each 4-hour chase period, we measured the amount of ${ }^{14} \mathrm{CO}_{2}$ and ASMs produced (Figure 5B). As with our earlier experiment (Figure $2 \mathrm{G}$ ), there was no difference in the amount of FA oxidation measured in control ASO- and apoB ASO-treated hepatocytes at the end of the 2-hour labeling period. We also observed no difference after the first 4-hour chase period. However, there was a significant increase in oxidation of ${ }^{14} \mathrm{C} \mathrm{OA}$ in the apoB ASO-treated hepatocytes, as compared with control cells, during each subsequent 4-hour chase period, starting at the 8-hour collection point (Figure $5 \mathrm{~B}$ ). When we added up all the ${ }^{14} \mathrm{C}$ OA that was oxidized over the entire 16-hour chase period, we found a significant increase in FA oxidation in the apoB ASO-treated hepatocytes as compared with control ASO-treated hepatocytes (Figure 5C). In one experiment, media from parallel wells were collected for lipid extraction, and the amount of ${ }^{14} \mathrm{C}$ TG that was secreted over the same time periods was measured (Figure 5D). As expected, we found significantly more ${ }^{14} \mathrm{C}$ TG secretion in the control ASO-treated hepatocytes compared with apoB ASO-treated cells during the 4-, 8-, and 12-hour chase periods. In fact, when the amount of ${ }^{14} \mathrm{C}$ TG secreted by the control cells was summed for the entire chase period, it was approximately equal to the amount of ${ }^{14} \mathrm{COA}$ that was oxidized by the apoB ASO-treated cells over the same time period. These results indicate that inhibition of the secretion of TG in apoB ASO-treated livers results in a compensation characterized by increased oxidation of FA via the autophagic pathway. This increase in autophagy-mediated FA oxidation enabled the apoB ASO-treated livers to maintain lipid homeostasis, thereby avoiding hepatic steatosis.

Given the finding of delayed but increased FA oxidation in apoB ASO-treated hepatocytes, we determined the levels of $\beta$-hydroxybutyrate in serum at 3 and 6 weeks of ASO treatments and found no differences among the 3 groups of mice (Supplemental Figure 10).

Inhibition of either lysosomal degradation or autophagy blocks FA oxidation in 6-week apoB ASO-treated hepatocytes. The delay in oxidation of FAs in newly synthesized (radiolabeled) lipids in apoB ASO-treated mice was consistent with the time required for transport of autophagosomes through the autophagic pathway to the lysosome $(23,24)$. As noted earlier, we observed, by immuno- fluorescence staining, increased LAMP2, a marker of lysosomes. To characterize the physiologic status of these lysosomes, we used LysoSensor to determine their $\mathrm{pH}$ in primary hepatocytes: there were no differences in the lysosomal acidification capacity across the 3 groups of mice at 6 weeks, indicating that the lysosomes present in greater numbers in the apoB ASO-treated livers were functioning normally (Supplemental Figure 11).

To confirm that the increased FA oxidation in hepatocytes from apoB ASO-treated mice was dependent on lysosomal degradation of lipid, we labeled the hepatocytes for 16 hours with ${ }^{14} \mathrm{C}$ OA, followed by a 4-hour chase, but treated half of the cells with $50 \mu \mathrm{M}$ chloroquine to block lysosomal activity (15). The primary hepatocytes from apoB ASO-treated mice once again showed an increase in oxidation of ${ }^{14} \mathrm{C} \mathrm{OA}$ in the nontreated cells as compared with hepatocytes from control ASO-treated mice (Figure 5E). This increase was blocked, however, by chloroquine treatment, indicating that the labeled lipid had to be hydrolyzed in the lysosome before being oxidized. While chloroquine treatment also significantly decreased FA oxidation in the control ASO-treated hepatocytes, this decrease was much smaller than that observed in the apoB ASO-treated cells. We also examined the role of the lysosome by inhibiting its functions with a combination of ammonium chloride and leupeptin and again observed a reduction of FA oxidation in apoB ASO-treated hepatocytes (Supplemental Figure 12).

To determine whether autophagy was involved in the increase in FA oxidation by the lysosome in the apoB ASO-treated mice, we used 3-methyladenine (3-MA) to block the formation of autophagosomes. While treatment with 3-MA was associated with a modest increase in the amount of ${ }^{14} \mathrm{C}$ OA oxidized in the control ASOtreated hepatocytes, 3-MA dramatically reduced the elevated level of FA oxidation in the apoB ASO-treated hepatocytes as compared with that detected in the nontreated cells (Figure 5F). This result demonstrated that autophagy is necessary for the increase in FA oxidation observed in the apoB ASO-treated livers.

If the increase in FA oxidation is blocked by chloroquine or 3-MA, then there should be an accumulation of TG in the apoB ASO-treated hepatocytes. To test this, we extracted lipid from the cells at the end of the chase period and measured the amount of ${ }^{14} \mathrm{C}$ TG that remained in the cells. With both chloroquine and 3-MA treatment, we found a significant increase in the amount of ${ }^{14} \mathrm{C}$-labeled $\mathrm{TG}$ remaining in those cells at the end of the experiment as compared with cells that had not received treatment (Figure 5G). Neither chloroquine nor 3-MA had any effect on the amount of ${ }^{14} \mathrm{C}$ TG remaining in the control ASO-treated hepatocytes. Furthermore, mice treated for 10 days with chloroquine $(60 \mathrm{mg} / \mathrm{kg} /$ day $)$ after 6 weeks of treatment with apoB ASO showed an increase in liver TG levels compared with mice receiving saline alone. Chloroquine had no effect on liver TG levels in mice treated with control ASO (Figure $5 \mathrm{H}$ ).

Delayed FA oxidation in mice treated for 6 weeks with apoB ASO occurs in the mitochondria. To determine whether increased FA oxidation in apoB ASO-treated mice occurred in mitochondria, we incubated primary hepatocytes from control ASO- and apoB ASO-treated mice with etomoxir, an inhibitor of carnitine palmitoyltransferase I that blocks mitochondrial FA oxidation (25). We found that etomoxir ablated the late increase in FA oxidation in apoB ASO-treated mice (Supplemental Figure 13). 
A
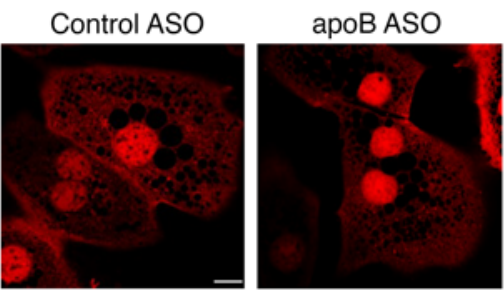

B
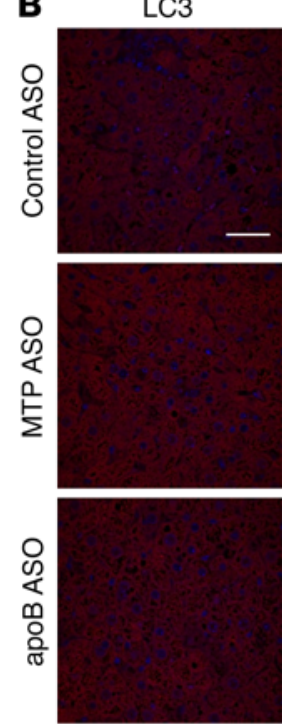

c

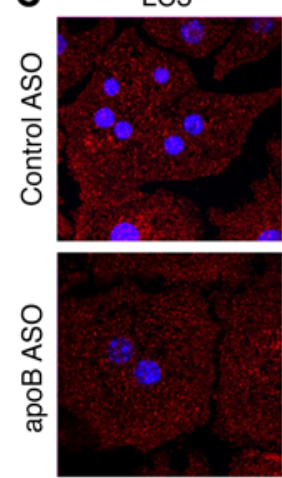

Calnexin
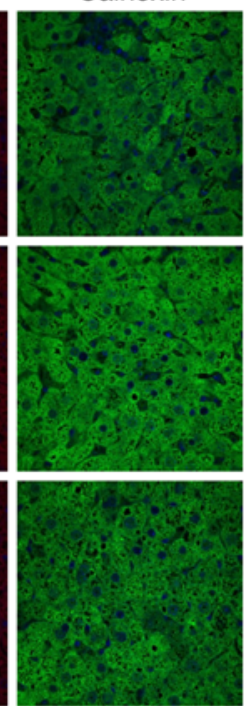

Calnexin

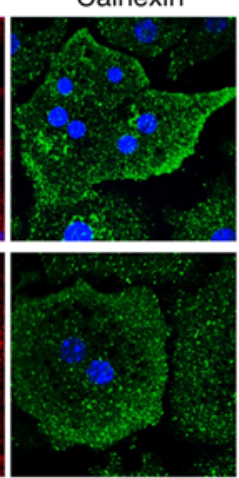

Merge

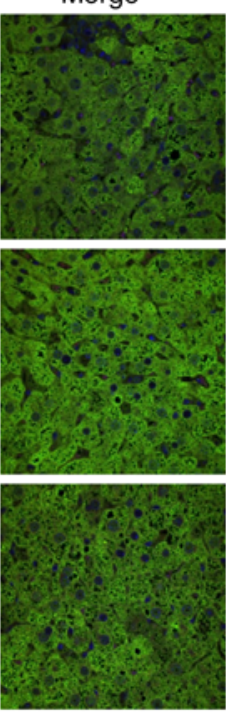

Merge
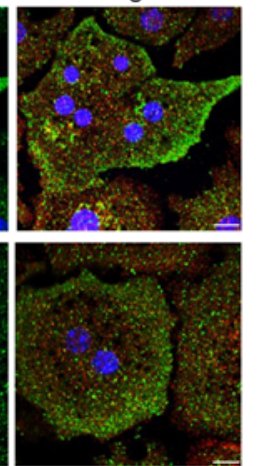

D

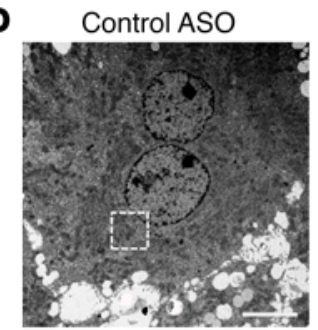

E

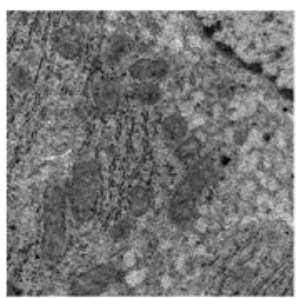

$\mathbf{F}$
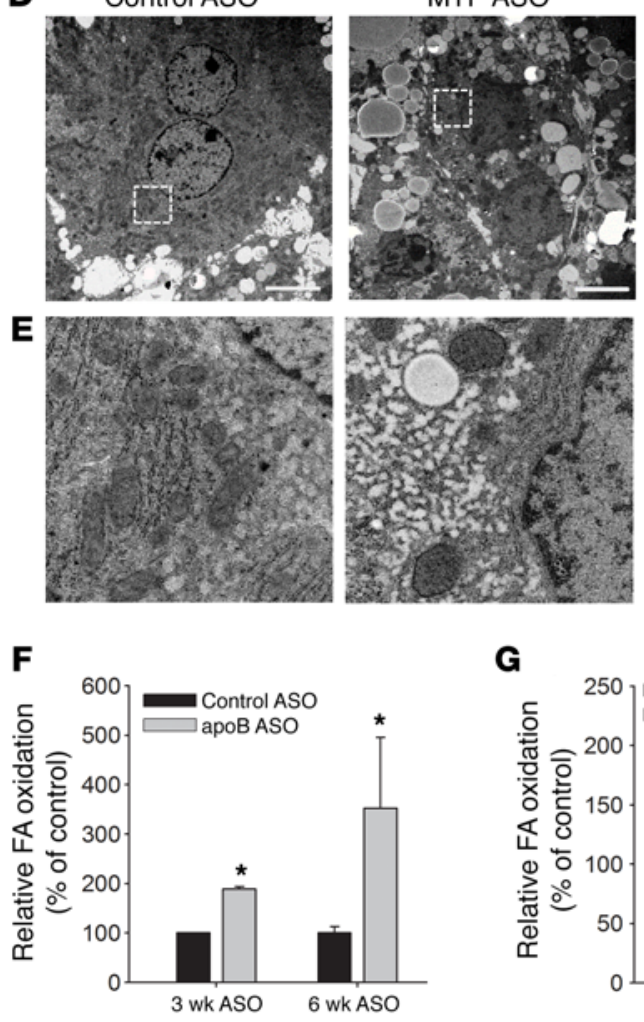

G
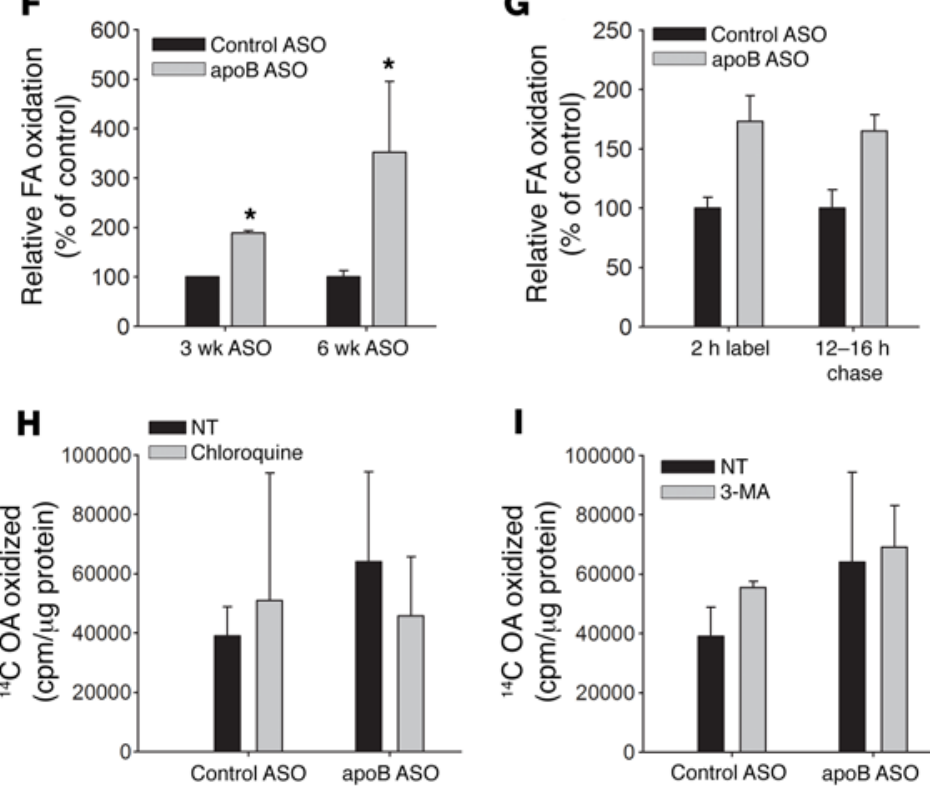

I

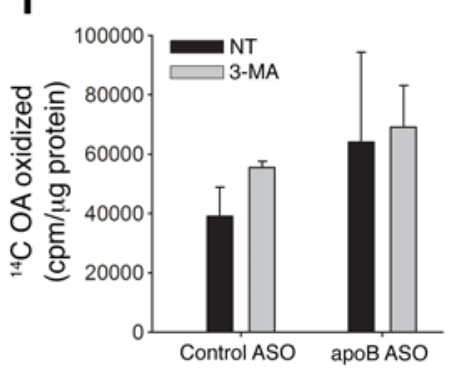

Figure 7. Three weeks of apoB ASO treatment does not cause a change in ER autophagy but does show an increase in lipid accumulation in the ER lumen. (A) Primary hepatocytes were stained with ER-Tracker Red for 30 minutes. $N=2$ mice; 3 images per mouse. Scale bar: $10 \mu$ m. (B) Liver sections were incubated with anti-LC3 Ab (red) and anti-calnexin Ab (green) and then stained with DAPI (blue). Scale bar: $20 \mu \mathrm{m}$. (C) Primary hepatocytes were incubated with anti-LC3 Ab (red) and anti-calnexin (green) and stained with DAPI (blue). $N=3$ mice per group; 3 images per mouse. Scale bars: $10 \mu$ m. (D) Representative EM images (original magnification, $\times 6,000$ ) of livers from mice treated for 3 weeks. Scale bars: $5 \mu$ m. (E) Insets of representative electron micrographs from $\mathbf{D}$ showing perinuclear areas with enlarged smooth ER in apoB ASO-treated mice. (F) Primary hepatocytes from mice treated for 3 or 6 weeks were labeled with ${ }^{14} \mathrm{C}$ OA for 16 hours and chased for 4 hours. ${ }^{14} \mathrm{CO}_{2}$ and ${ }^{14} \mathrm{C}$ ASM were measured and normalized to cell protein levels. $N=9$ wells from 3 mice ( 3 weeks); $N=15$ wells from 5 mice ( 6 weeks). ${ }^{*} P<0.05$, by Student's $t$ test, for apoB ASO versus control ASO for each time period. (G) Primary hepatocytes were labeled with ${ }^{14} \mathrm{C} \mathrm{OA}$ for 2 hours, and chase media were added to the hepatocytes for 12 hours and then changed for another 4 hours. The amount of ${ }^{14} \mathrm{CO}$ and ${ }^{14} \mathrm{C}$ ASMs produced during labeling and the 12 - to 16 -hour chase was measured. $N=9$ wells from 3 mice per group. (H and I) Hepatocytes were labeled with ${ }^{14} \mathrm{C}$ OA for 16 hours and then chased for 4 hours with or without $50 \mu \mathrm{M}$ chloroquine or 5 mM $3-\mathrm{MA}$, after which ${ }^{14} \mathrm{CO}$, and ${ }^{14} \mathrm{C} \mathrm{ASMs}$ were measured and normalized to cell protein levels. $N=9$ wells from 3 mice per group. All values represent the mean \pm SD.

Knockdown of apoB using ASO in C57BL/6J mice. We repeated the experiments in $\mathrm{C} 57 \mathrm{BL} / 6 \mathrm{~J}$ mice using control ASO and apoB ASO for 6 weeks. We observed a significant decrease in both TG and apoB secretion in apoB ASO-treated mice but found no significant differences in hepatic TG levels among the mice treated with apoB ASO or control ASO (Supplemental Figure 14, A-C). ER auto- phagy, accompanied by increased FA oxidation, was blocked by treatment with chloroquine (Supplemental Figure 14, D-G). Treatment of both C57BL/6J and apobec-1-KO mice with a small-molecule inhibitor of MTP (BMS-212122) resulted in a suppression of VLDL secretion and steatosis similar to that observed with MTP ASO (data not shown). 
A
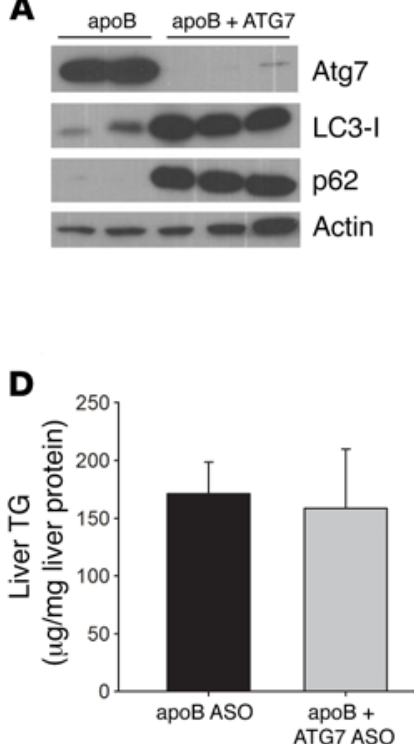

G

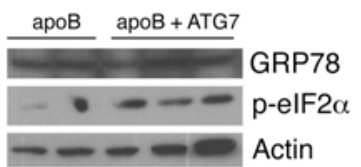

B

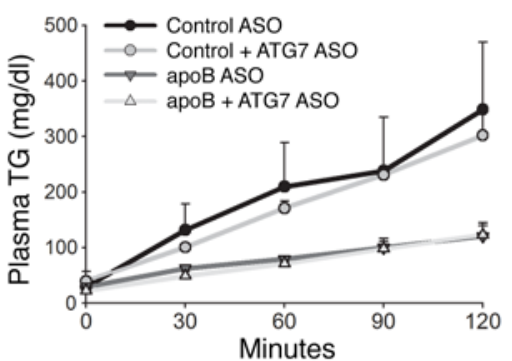

E

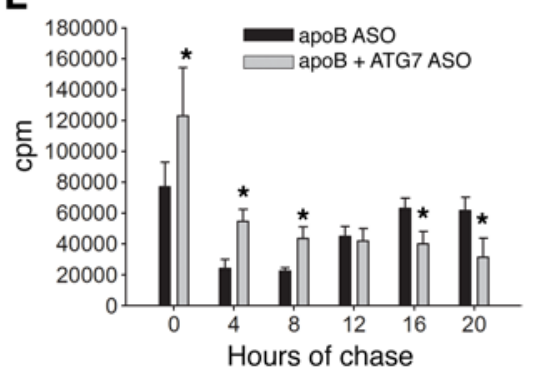

H

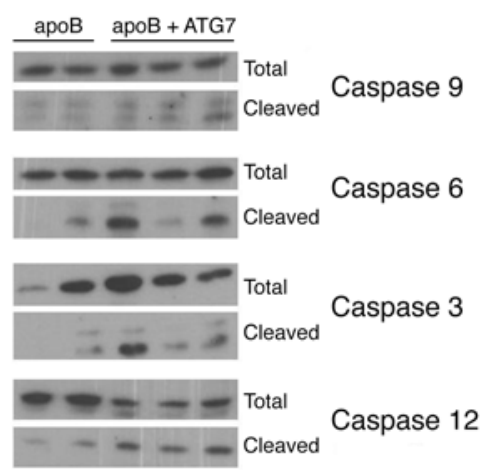

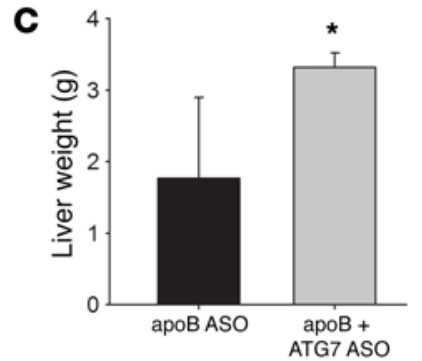

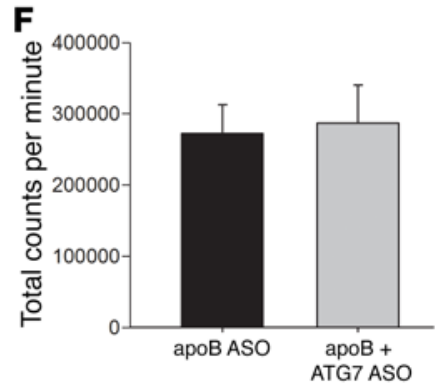

Figure 8. ATG7 ASO inhibits autophagy in apoB ASO-treated mice but has no effect on either TG secretion or liver TG, despite an increase in liver weight. Increased ER stress and apoptosis were also evident. Apobec-1-KO mice were treated with apoB ASO for 6 weeks, and either control or ATC7 ASO was added for the final 3 weeks. (A) Liver homogenate was run on an SDS-PAGE and immunoblotted for ATC7, LC3, p62, and actin. $N=5-6$ livers per group. (B) Triton WR1339 was injected i.v., blood samples were obtained every 30 minutes over the next 120 minutes, and plasma TG levels were measured. $N=2-3$ mice per group. (C) Livers were weighed at the time of euthanization. $N=5-6$ livers per group. ${ }^{*} P<0.05$, by Student's $t$ test, for apoB plus ATC7 ASO versus apoB ASO control. (D) Liver lipids were extracted, and TG was measured enzymatically. $N=5-6$ livers per group. (E) Primary hepatocytes were labeled with ${ }^{14} \mathrm{C}$ OA for 2 hours, and then unlabeled chase media were added and collected every 4 hours over a 16 -hour period. $N=6$ wells from 2 mice per group. ${ }^{*} P<0.05$, by Student's $t$ test, for apoB plus ATC7 ASO versus apoB ASO control. (F) The total amount of ${ }^{14} \mathrm{C}$ OA oxidized over the 16 -hour time points was summed for each group of mice. $N=6$ wells from 2 mice per group. (G) Liver homogenate was run on a 10\% SDS-PACE and immunoblotted for the ER stress markers GRP78, p-eIF2 $\alpha$, and actin. $N=5-6$ livers per group. (H) Liver homogenates were run on $8 \%$ or 12\% SDS-PACE and immunoblotted for various markers of apoptosis, either total or cleaved. $N=5-6$ livers per group. All values represent the mean \pm SD.

Mice treated for 3 weeks with apoB ASO have increased hepatic TG levels and evidence of ER stress, but no increased autophagy. As previously noted, we did not observe an increase in ER stress after 6 weeks of apoB ASO treatment, a surprising result because of the assumed increase in lipid trapped in the lumen of the ER in this model of inhibited VLDL secretion. Therefore, we examined the liver phenotype of apoB ASO-treated mice after just 3 weeks of treatment. Knockdowns of hepatic MTP and apoB gene expression were comparable to those seen after 6 weeks of ASO treatment (data not shown). Secretion of VLDL-TG and apoB was also decreased to a degree similar to that seen after 6 weeks of each ASO treatment (Figure 6, A and B), as were plasma cholesterol levels (Supplemental Figure 2). However, in contrast to the effect of 6 weeks of apoB ASO treatment, we observed a significant increase in hepatic TG levels after 3 weeks of apoB ASO com- pared with levels detected with control ASO treatment (Figure 6C). Furthermore, we found no significant difference in hepatic TG levels between mice receiving apoB ASO and those receiving MTP ASO treatment for 3 weeks. This was confirmed by oil red O staining on liver sections, which showed that apoB ASO- and MTP ASO-treated livers had LDs that were similar in size and number (Figure 6D). Concomitant with increased hepatic steatosis, and in contrast to our findings after 6 weeks of apoB ASO treatment, we observed increases in markers of ER stress, including GRP78 (Figure 6E; densitometry in Supplemental Figure 15) and XBP1 splicing (Supplemental Figure 15), in the apoB ASO-treated livers compared with both control ASO- and MTP ASO-treated livers. Also contrary to 6 weeks of treatment, we found no difference in the levels of LC3-II as measured by immunoblotting, with or without lysosomal inhibitors, in primary hepatocytes isolated after 3 


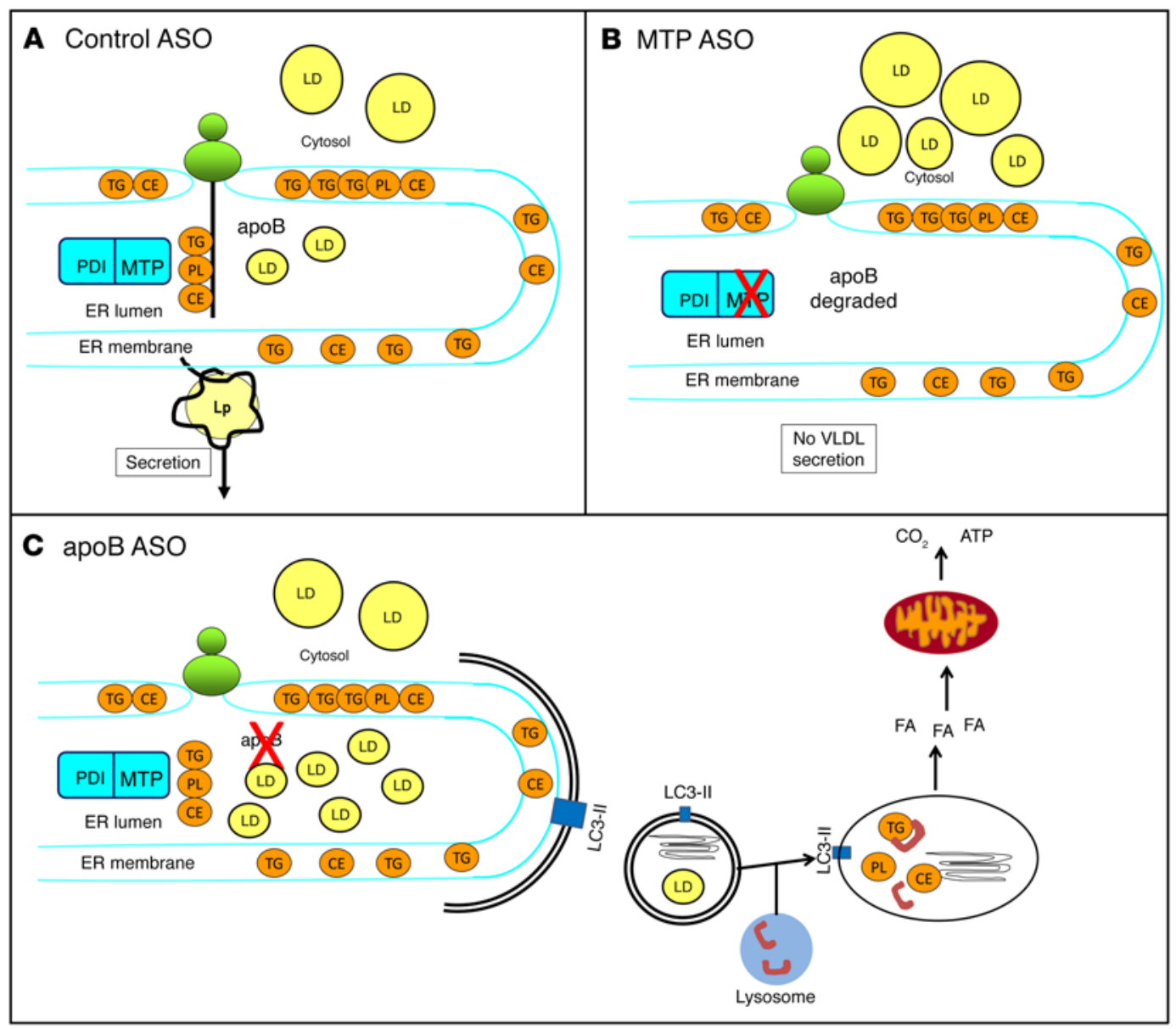

Figure 9. ER autophagy-mediated FA oxidation prevents steatosis when apoB synthesis is inhibited but MTP is active. (A) In control ASO-treated mice, MTP lipidates nascent apoB as it translocates across the ER. Further continuous lipidation or fusion with ER luminal droplets results in the formation of VLDL, which exits the ER on the way to secretion. Some newly synthesized lipids are transferred to cytosolic LDs in control ASO-treated mice. (B) In MTP ASO-treated mice, the transfer of lipid from the ER membrane into the lumen of the ER is prevented, apoB protein is cotranslationally degraded, VLDL assembly and secretion is blocked, and newly synthesized lipids can only be transferred into cytosolic LDs. (C) In apoB ASO-treated mice, lipid transferred by MTP into the lumen of the ER accumulates in the absence of apoB. This stimulates the formation of double-membrane autophagosomes that engulf lipid-filled regions of the ER. The autophagosomes fuse with lysosomes, where lipids, including TC, are hydrolyzed by acidic lipases. The FA generated by lipolysis become available for oxidation in mitochondria. PDI, protein disulfide isomerase ; Lp, lipoprotein.

weeks of either control ASO or apoB ASO treatment (Figure 6F). We also observed no difference in immunofluorescence staining for LC3 after apoB ASO treatment compared with control ASO or MTP ASO treatment in either liver sections or primary hepatocytes (Figure 6, G and H). Furthermore, p62 levels did not differ across the 3 groups of mice (Supplemental Figure 8).

Three weeks of apoB ASO treatment does not induce ER autophagy. Staining with ER-Tracker Red in primary hepatocytes treated with ASO for 3 weeks showed no difference between control and apoB knockdown (Figure 7A). Additionally, there was no increase in the colocalization of LC3 and calnexin in either the apoB ASOtreated liver sections or primary hepatocytes (Figures 7, B and C), indicating that there was no increase specifically in ER autophagy. Finally, electron micrographs showed no increase in autophagosomes in the livers treated with apoB ASO for 3 weeks compared with both the control ASO- and MTP ASO-treated livers (Figure 7D). Although we hypothesized that TG trapped in the ER lumen was the signal for ER stress and, later, for autophagy, we were unable to see colocalization of BODIPY and calnexin in primary hepatocytes after 3 weeks of apoB ASO treatment (Supplemental Figure 16). We did, however, observe marked increases in the smooth ER content of liver cells from mice treated with apoB ASO for 3 weeks (Figure 7E and Supplemental Figure 17).

To better characterize the changes in the ER observed by EM at 3 and 6 weeks, we isolated ER (26) from control ASO- and apoB ASO-treated livers and determined lipid levels by mass spectrometry. ER TG and ER diglyceride (DG) levels fell dramatically between 3 and 6 weeks. During the same interval, phosphatidylcholine (PC) levels rose slightly but significantly, whereas phosphatidylethanolamine (PE) increased by almost 3-fold (Supplemental Figure 18). These changes in levels of key PLs in the ER might be a reflection of the presence of ER stress at 3 weeks, which has been shown by Fu et al. (27) to be associated with an increased ratio of $\mathrm{PC}$ to $\mathrm{PE}$. Those investigators also showed that reversal of this ratio led to a return to ER homeostasis, which we observed to be temporally associated with an increase in PE at 6 weeks. The 
$\mathrm{PC} / \mathrm{PE}$ ratios did not differ between control ASO- and apoB ASOtreated mice at 3 weeks, but the $\mathrm{PC} / \mathrm{PE}$ ratio was markedly lower in the apoB ASO-treated group at 6 weeks (data not shown). These results are consistent with the increase in PE in the ER at 6 weeks (Supplemental Figure 18). On the basis of the work of Fu et al. (27), we also determined hepatic expression of phosphatidylethanolamine $N$-methyltransferase (PEMT), the enzyme that converts PE to $\mathrm{PC}$ and found it to be similar in all 3 groups of mice at both time points (Supplemental Figure 19). It is interesting to note that covalent binding of PE to LC3-I converts the latter to the active LC3-II form at the initiation of autophagy.

Activation status of AMPK and mTOR differs at 3 and 6 weeks, consistent with a shift from ER stress to ER autophagy. The shift from ER stress to autophagy between 3 and 6 weeks of apoB ASO treatment suggested changes in the activity of AMPK, which has been implicated as a trigger of autophagy, and mTOR, a key regulator of autophagy (28). Phosphorylation of AMPK tended to be increased at 3 weeks, but not at 6 weeks, in apoB ASO-treated mice (Supplemental Figure 20), whereas phosphorylation of mTOR was significantly increased at 3 weeks, but was not different between groups at 6 weeks.

Activation of CaMKII did not change between 3 and 6 weeks of apoB ASO treatment. The shift from ER stress to ER autophagy between 3 and 6 weeks also suggested changes in ER calcium homeostasis. Calcium/calmodulin-dependent protein kinase II (CaMKII) is phosphorylated, and thereby activated, when ER calcium homeostasis is disrupted, and cytosolic calcium increases as a result. We did not find differences in CaMKII phosphorylation among the 3 groups of mice at either 3 or 6 weeks of ASO treatment (Supplemental Figure 21).

Three weeks of apoB ASO treatment increases FA oxidation, but the increase is not delayed. We measured FA oxidation in primary hepatocytes isolated from mice treated for 3 weeks with control or apoB ASO by once again labeling the hepatocytes with ${ }^{14} \mathrm{C}$ OA for 16 hours, followed by a 4-hour chase. We observed a significant increase (90\%) in the amount of ${ }^{14} \mathrm{CO}_{2}$ and ${ }^{14} \mathrm{C}$ ASMs produced in the apoB ASO group as compared with that seen in the control ASO group (Figure 7F). Importantly, the increase in FA oxidation after 3 weeks of apoB ASO treatment was neither as great as that seen after 6 weeks of apoB ASO treatment (Figure 7G), nor was it delayed (Figure 7F). The modest nature of this increase in FA oxidation was paralleled, as noted earlier, by an absence of differences in serum $\beta$-hydroxybutyrate levels across the 3 groups of mice (Supplemental Figure 10). These latter observations are consistent with the finding of an increase in liver TG levels after 3 weeks of apoB ASO treatment that was not seen at 6 weeks. To confirm that neither autophagy nor lysosomes played a role in the increased FA oxidation observed after 3 weeks of administration of apoB ASO, primary hepatocytes were treated with either 3-MA or chloroquine prior to measurement of FA oxidation. There was no significant decrease in FA oxidation with either treatment as compared with nontreated apoB ASO or control ASO-treated hepatocytes (Figures 7, H and I).

Inhibition of autophagy using ATG7 ASO causes apoptosis in apoB ASO-treated livers. Increased lipid accumulation after 3 weeks of apoB ASO treatment with evidence of ER stress, but not autophagy, led us to inhibit autophagy starting after 3 weeks of apoB ASO treat- ment to see whether even greater hepatic lipid accumulation would be observed after 6 weeks of treatment. After 3 weeks of apoB ASO treatment, half of the mice continued to receive apoB ASO alone for the final 3 weeks, while the other half were also injected with ASO against ATG7, a protein that is necessary for the formation of LC3-II and therefore essential for initiation of autophagy. ATG7 ASO treatment resulted in a complete absence of LC3-II protein and accumulation of p62, indicative of decreased autophagy (Figure 8A), without affecting TG secretion in either group (Figure 8B). apoB-ATG7 ASO livers were significantly larger than the apoB ASO livers, but liver TG levels were similar (Figure 8, C and D).

Primary hepatocytes were isolated, and we repeated the timecourse FA oxidation study. When the total amount of ${ }^{14} \mathrm{C}$ OA oxidized over the entire chase period was summed, we found no significant difference between the apoB ASO- and apoB-ATG7 ASO-treated groups. However, although we replicated the previously observed delayed FA oxidation in the apoB ASO-treated hepatocytes, labeled FA was oxidized during the early time points in the apoB-ATG7 ASO-treated group, indicating that the lipid being oxidized did not traverse the autophagic pathway (Figure 8, E and F).

Furthermore, in contrast to apoB ASO treatment alone, inhibition of autophagy during the last 3 weeks of apoB ASO treatment was associated with continued ER stress and an increase in several markers of apoptosis (Figures 8, G and $\mathrm{H}$ ). Thus, increased autophagy of the ER in the apoB ASO-treated hepatocytes between 3 and 6 weeks of treatment was protective; failure to upregulate ER autophagy after 3 weeks of apoB ASO treatment, in this instance because of suppression of ATG7 synthesis, resulted in the persistence of ER stress and the UPR, ultimately triggering apoptosis and signs of cell death by 6 weeks. In association with the evidence for apoptosis and hepatotoxicity, we observed increased oxidation of the lipids that were not secreted, but this did not occur via autophagy.

\section{Discussion}

Hepatic lipid homeostasis is maintained by a balance of inputs, including uptake of circulating albumin-bound FA, uptake of FA carried in TG by chylomicron and VLDL remnants, and DNL, and outputs, including VLDL secretion and FA oxidation (29). Any increase in hepatic lipid uptake or DNL that is not balanced by an increase in FA oxidation or VLDL secretion will result in accumulation of hepatic TG, known as steatosis. The clinical relevance of the loss of hepatic lipid homeostasis is evident from both the very high prevalence of nonalcoholic fatty liver disease (NAFLD) associated with obesity and insulin resistance and the demonstrated progression of NAFLD to end-stage liver disease (30). By comparing the effects on hepatic steatosis of inhibiting either MTP or apoB synthesis, we have generated data that (a) add ER autophagy to the previously well-characterized pathways for maintaining hepatic lipid homeostasis, including lipophagy (31); (b) provide insights into a rapidly expanding area of work linking ER stress to autophagy (32); (c) suggest new approaches to studying the partitioning of newly synthesized hepatic lipids between the ER and cytosolic LDs (33); and (d) offer the possibility that inhibition of VLDL secretion, a potentially powerful target for lowering all atherogenic apoB lipoproteins, might be possible with only minimal or modest increases in steatosis. 
Autophagy is used by cells and organs to generate energy during nutrient deprivation and to maintain cellular homeostasis of proteins and organelles (34). However, impaired autophagy, including the recently characterized lipophagic pathway (15), can contribute to hepatic steatosis and insulin resistance $(21,31$, 35). We used apoB ASO to create a unique perturbation, in which MTP-mediated transfer of newly synthesized lipids, particularly TG, from the ER membrane into the ER lumen is intact but is not followed by apoB-mediated transport of those lipids to the downstream components of the secretory pathway and out of the hepatocyte. Our results demonstrate clearly that the response to this discordance between lipid movement into the ER lumen (36) and the absence of VLDL assembly was ER-specific autophagy: increased ER with abnormal aggregates, increased LC3-II that colocalized with calnexin, and the absence of colocalization of LC3-III with markers of mitochondria or LDs. Of note, autophagy of the ER did not disturb the secretion of albumin or apoA-I, suggesting that this autophagy was restricted to regions where, we assume, lipid was accumulating. Importantly, ER autophagy alone would not have been adequate to maintain lipid homeostasis; our demonstration of delayed but increased FA oxidation provided crucial support for our model. That the increased FA oxidation occurred via the autophagic pathway was demonstrated by the effects of chloroquine, ammonium chloride/leupeptin, and 3-MA: each treatment inhibited FA oxidation in primary hepatocytes from apoB ASO-treated mice, resulting in the accumulation of TG. We also showed that FA oxidation occurred via mitochondria. In vivo chloroquine treatment also increased whole-liver TG levels in apoB ASO-treated mice. The lack of an association of autophagosomes with LDs in apoB ASO-treated mice indicated that we were not seeing an increase in lipophagy, as described by Singh et al. (15). The absence of lipophagy in either apoB ASP- or MTP ASO-treated mice was surprising, but might have been related to our use of a diet high in total and saturated fats (15).

Our studies after 3 weeks of apoB ASO treatment indicated that ER stress, which we expected but did not observe at 6 weeks, preceded the appearance of ER autophagy. ER stress is caused by various perturbations, including excess hepatic lipids, and is characterized by misfolding of ER proteins, leading to upregulation of the UPR (37), which is associated with increased lipid synthesis (38) and inhibition of VLDL secretion (20). Previous studies have demonstrated that LDs without apoB are present in the lumen of the smooth ER in normal livers (39) and accumulate in the secretory pathway of the intestines of mice lacking apoB but with normal MTP levels in that organ (40). We believe that this was occurring in the liver of our apoB ASO-treated mice, as indicated by expansion of the smooth ER after 3 weeks of apoB ASO treatment, although ER expansion is a concomitant of ER stress in general (41). Further studies will be required to identify the pathway linking the accumulation of unsecreted lipids in the ER to ER stress, particularly regarding the role of protein misfolding. Our results indicate, however, that ER stress, stimulated by a continuous flow of newly synthesized lipids that are trapped in the lumen of the ER, triggers specific ER autophagy. Changes in AMPK and mTOR phosphorylation between 3 and 6 weeks are consistent with the temporal change from ER stress to ER autophagy that we observed. The critical role of the latter pathway is clearly demon- strated by the (a) disappearance of ER stress by 6 weeks when ER autophagy was active; (b) marked reductions in ER levels of TG and DG by 6 weeks compared with levels at 3 weeks; and (c) persistence of ER stress and the appearance of several markers of apoptosis, including cleaved caspase 12 , which has been shown to be specifically induced by ER stress (42), when initiation of autophagy was blocked by ATG7 ASO treatment. The increased FA oxidation we observed when autophagy was blocked and which, like autophagy, prevented steatosis in the apoB ASO- and ATG7 ASOtreated mice, very likely resulted from lipids released directly from "sick" ER; we will attempt to better characterize this observation in future studies. Our model should, however, lead to a better definition of the link between ER stress and autophagy that, despite being the focus of a significant number of publications in the past several years, remains incompletely characterized (32, 43-45). In particular, whether ER autophagy occurs by nonselective or selective autophagy and whether micro- or macroautophagy or both are involved are questions in an ongoing area of intense investigation (46-49). Our findings that the increased FA oxidation is blocked by treatment with 3-MA, together with the loss of delayed FA oxidation in mice treated with ATG7 ASO, support the idea of selective macroautophagy as the pathway stimulated by inhibition of VLDL assembly and secretion. Recent work identifying key proteins involved in the trafficking and turnover of the ER, such as ATG39/40 (50) and members of the related FAM134 family of reticulon proteins (51), can be the focus of future experiments using our model of ER autophagy.

Movement of newly synthesized lipids from the ER membrane to either cytosolic droplets or the lumen of the ER, where they can be incorporated into VLDL, has stimulated significant interest for several decades among scientists studying hepatic lipid metabolism $(52,53)$. Although studies of the cell and molecular biology of LDs have expanded dramatically in the past 10 years (33), basic questions, such as what determines the direction that newly synthesized ER lipids take, whether luminal TG that is ultimately secreted in VLDL has to first move to an LD after synthesis and undergo lipolysis before returning to the ER, and whether ER luminal TG can move back to the ER membrane and then to LDs, remain fully or partially unanswered. Our use of ASOs against MTP and apoB has provided some insights into these questions. For example, if ER luminal lipids/TG can move to the cytosol when the secretory pathway is unavailable, we should have seen as much steatosis with apoB ASO as with MTP ASO treatment, and no autophagy. Preliminary studies in which we have administered both MTP ASO and apoB ASO resulted in the phenotype observed in mice treated only with MTP ASO, i.e., there was significant steatosis and no increase in autophagy. This result indicates that autophagy only occurs in our model when ER membrane lipid can be transferred to the ER lumen. Our results also raise questions regarding the long-held model in which LD TG is the source of ER luminal TG that is secreted in VLDL; if this were the case, then ER autophagy could have targeted cytosol-derived lipids (from LD turnover) for oxidation via autophagy, possibly reducing hepatic $\mathrm{TG}$ in apoB ASO-treated mice to levels even below those seen in the control ASO-treated mice. We believe that by using these ASOs, along with others, such as ASOs against diacylglycerol $O$-acyltransferases 1 and 2 (DGAT1 and DGAT2), 
we will be able to better characterize the pathway of newly synthesized TG between the cytosol and ER.

Finally, our results, which included a significant reduction in plasma cholesterol levels in the apoB ASO-treated mice, raise the possibility that, by increasing autophagy, particularly ER autophagy, we may be able to reduce the secretion of VLDL and plasma levels of atherogenic lipoproteins without causing hepatic steatosis. If inhibition of apoB synthesis was coupled with stimulation of autophagy, treatments with drugs that block VLDL secretion might not be limited in dose or potency by hepatic steatosis. In a similar fashion, inhibition of the movement of newly synthesized TG from the ER to the LD, so that the TG would be forced to move into the ER lumen, with secondary stimulation of autophagy, might also produce lower plasma lipoproteins without hepatic steatosis.

In summary, knockdowns of MTP and apoB were equally efficacious at inhibiting VLDL secretion, but only the knockdown of MTP was associated with hepatic lipid accumulation. The attenuated hepatic lipid storage phenotype observed with the knockdown of apoB was attributable to an increase in autophagy that targeted the lipid trapped in the ER to the lysosome. FA generated by lysosomal lipases were released and oxidized by mitochondria. This schema is depicted in Figure 9.

\section{Methods}

See Supplemental Methods for additional details.

Animal care apobec-1-KO mice (provided by Nicholas Davidson, Washington University, St. Louis, Missouri, USA) and C57BL/6 mice (The Jackson Laboratory) were housed 3-4 mice per cage in a climate-controlled room with a 12-hour light/12-hour dark cycle. Animals were fed ad libitum a Western-type HFD containing $4.5 \mathrm{kcal} / \mathrm{g}$ of food with $21 \%(\mathrm{w} / \mathrm{w})$ fat (polyunsaturated/saturated $=0.07)$ and $0.15 \%(w / w)$ cholesterol. The HFD consisted of $42 \%$ of calories from fat (29\% of which were from saturated fat) and $30.5 \%$ from sucrose (Harlan Teklad; TD88137).

ASO studies. Mice (12-24 weeks old) on a HFD for 3 or 6 weeks were injected i.p. biweekly with $50 \mathrm{mg} / \mathrm{kg}$ ASO directed against apoB (ISIS 147764), MTP (ISIS 144477), or scrambled (control; ISIS 299705) messages for 6 weeks. The ASOs were 20-mer phosphorothioate oligonucleotides containing 2'-O-methoxyethyl groups at positions 1-5 and 15-20 and were provided by Ionis Pharmaceuticals. At the end of studies, animals were anesthetized with ketamine/xylene and euthanized by cervical dislocation. Livers were either quickly excised, flash frozen, and kept at $-80^{\circ} \mathrm{C}$ until analyzed, or primary hepatocytes were isolated.

Liver lipids. Approximately $100 \mathrm{mg}$ of liver tissue was homogenized in $3 \mathrm{ml}$ of PBS. The homogenizer generator was rinsed with ethanol and water between samples. After homogenization, the lipids were isolated by Folch extraction (final chloroform/methanol/water solution $=8: 4: 3$ ). The organic layer was carefully evaporated with liquid nitrogen to dryness. The sample was reconstituted in $1 \mathrm{ml}$ of $15 \%$ Triton X-100 in chloroform, which was evaporated with nitrogen and resuspended in $1 \mathrm{ml}$ of water. TG and cholesterol concentrations were measured enzymatically (Wako Chemicals).

Immunofluorescence staining. Paraffin-embedded 5- $\mu$ m liver sections were deparaffinized and rehydrated. Antigen retrieval was performed by boiling with $10 \mathrm{mM}$ sodium citrate. Primary hepatocytes were plated onto collagen-coated glass-bottomed 35-mm plates. After 24 hours, the cells were fixed with $10 \%$ formalin for $30 \mathrm{~min}$ - utes and then washed 3 times with PBS. The section or hepatocytes were blocked in PBS with 5\% normal serum and 0.3\% Triton X-100 for 1 hour. Incubation buffer with primary $\mathrm{Ab}$ was added overnight. The primary Ab used was rabbit anti-LC3B Ab (Cell Signaling Technology; catalog 2775) alone or rabbit anti-LC3B Ab with either goat anti-calnexin Ab (Abcam; catalog ab93355); rat anti-LAMP2 Ab (University of Iowa; Developmental Studies Hybridoma Bank product GL2A7); chicken anti-ADRP Ab (Abcam; catalog ab37516); or goat anti-VDAC Ab (Abcam; catalog ab37459). Sections were incubated with a secondary $\mathrm{Ab}$ for 1 hour. We used the secondary Ab Alexa Fluor 555-conjugated anti-rabbit IgG Ab alone or with Alexa Fluor 647-conjugated anti-goat IgG Ab; Alexa Fluor 647-conjugated anti-chicken IgG Ab; or Alexa Fluor 647-conjugated anti-rat IgG Ab. Prolong Gold Anti-fade Reagent with DAPI (Invitrogen, Thermo Fisher Scientific) was added onto coverslips, allowed to cure for 24 hours, and then sealed. Images were visualized using a NikonA1RMP confocal microscope (original magnification, $\times 600$ ).

Immunoblots. Snap-frozen liver or cells were homogenized in lysis buffer containing protease inhibitors. Equal amounts of protein extract were separated by gel electrophoresis on appropriate percentage SDSPAGE gels and transferred to polyvinylidene fluoride membranes. Membranes were incubated with primary Abs overnight and then with species-specific HRP-conjugated secondary Abs. Protein bands were visualized using SuperSignal West Pico Chemiluminescent Substrate (Thermo Fisher Scientific). LC3 (catalog 2775), an Apoptosis Sample Kit (catalog 9915); ATG7 (catalog 2631); p-mTOR (Ser2448) (catalog 2971); total mTOR (catalog 2983); p-AMPKa (Thr172) (catalog 2535); and total AMPK $\alpha$ (catalog 5831) were all purchased from Cell Signaling Technology. GRP78 (Stressgen; catalog SPA-826); p-eIF2 $\alpha$ (Stressgen; catalog BML-SA405); p62 (Abnova; catalog H00008878-M01); apoA1 Ab (Thermo Fisher Scientific; catalog PA5-19784); ATP-binding cassette subfamily A (ABCA1) (Novus Biologicals; catalog 400105); SR-BI (Novus Biologicals; catalog 400-101); CaMKII alpha/beta [p Thr286, p Thr287] (Novus Biologicals; catalog NB110-96869); and actin (1:10,000; Sigma-Aldrich; catalog A5441) were also used.

FA oxidation measured in primary hepatocytes. Two hours after the plating of primary hepatocytes in 6-well plates, the media were changed to DMEM with $1.5 \% \mathrm{BSA}, 0.1 \mathrm{mM} \mathrm{OA}$, and ${ }^{14} \mathrm{C}$ OA $(1 \mu \mathrm{Ci} /$ $\mathrm{ml})$. The cells were incubated with the labeled media for 2 hours. In some experiments, the labeled media were collected for FA oxidation measurement, while in others, the media were changed to DMEM with $1.5 \%$ BSA only for repetitive 4 -hour chases up to 16 hours before collection for FA oxidation measurement. In other experiments, cells were incubated with labeled media for 16 hours and then chased with DMEM with $1.5 \%$ BSA for 4 hours, after which the media were collected for FA oxidation measurement. In some experiments, either 50 $\mu \mathrm{M}$ chloroquine, $5 \mathrm{mM}$ 3-methyladenine, $20 \mathrm{mM}$ ammonium chloride plus $100 \mu \mathrm{M}$ leupeptin, or $200 \mu \mathrm{M}$ etomoxir was added. At the end of the labeling or chase period, the media were transferred to $25-\mathrm{ml}$ sealed Erlenmeyer flasks. Lipid oxidation was stopped with the addition of $200 \mu \mathrm{l}$ of $70 \%$ perchloric acid to the bottom of the flask, driving the bicarbonate into $\mathrm{CO}_{2} \cdot{ }^{14} \mathrm{CO}_{2}$ was captured on a piece of $\mathrm{KOH}$ soaked filter paper. After incubating the filter paper in the flask for 1 hour at room temperature, the filter paper was analyzed for ${ }^{14} \mathrm{C}$ activity by liquid scintillation counting of ${ }^{14} \mathrm{CO}_{2}$. The media remaining in the flask were collected and a fraction counted for ${ }^{14} \mathrm{C}$ as a measure of ASM production. After the labeled and chase media were removed at 
the end of the experiment or time point, lipid was extracted from the cells by adding $2 \mathrm{ml}$ of 3:2 hexane/isopropanol directly to the 6-well plate for 2 hours. The hexane/isopropanol was collected, and 2 additional extractions with $1 \mathrm{ml}$ of hexane/isopropanol were performed. The lipid extract was dried under $\mathrm{N}_{2}$ gas, resuspended in hexane, and spotted on silica 60 TLC plates. The lipid was separated using a mobile phase of hexane/diethyl ether/acetic acid (70:30:1) and visualized using iodine. The PL, TG, FA, and cholesterol ester (CE) spots were scraped, and the ${ }^{14} \mathrm{C}$ counts in each spot were measured using a beta counter (Beckman Coulter). Total protein was recovered by solubilizing the remaining cells in the 6-well plate in $0.1 \mathrm{~N} \mathrm{NaOH}$ and measured using bicinchoninic acid (BCA) assay (Pierce, Thermo Fisher Scientific). Lipid counts were then normalized to total cell protein.

Statistics. Data are presented as the mean \pm SD. Differences in the mean values between 2 groups were assessed using a 2-tailed Student's $t$ test. Differences in mean values among more than 2 groups were assessed by ANOVA. A $P$ value of less than 0.05 was considered statistically significant. When ANOVA was significant, we used a Tukey's post test for individual comparisons.

Study approval. All animal procedures were approved by the IACUC of Columbia University.

\section{Author contributions}

DMC, TT, TF, AHO, GDP, RBC, KR, SG, JL, and HNG are responsible for the work described in this article. DC, TT, and HNG were responsible for conception, design, acquisition, analysis, statistics, interpretation, as well as, drafting and revising the manuscript. TF was responsible for conception, acquisition, analysis, interpretation, as well as, drafting and revising the manuscript. AHO was responsible for design, acquisition, analysis, statistics, interpretation, as well as, drafting and revising the manuscript. GDP and $\mathrm{RBC}$ were responsible for design, acquisition, analysis, interpretation, and revising the manuscript. KR was responsible for design, acquisition, analysis, statistics, and revising the manuscript. SG was responsible for design, acquisition, analysis, statistics, interpretation, and revising the manuscript. JL was responsible for acquisition, analysis, statistics, interpretation, and revising the manuscript. All authors provided final approval of the version to be published. All authors are accountable for all aspects of the work and for ensuring that questions related to the accuracy or integrity of any part of the work are appropriately investigated and resolved.

\section{Acknowledgments}

The authors thank Kristy Brown of the Electron Microscopy Facility for her assistance with the sample preparation and Theresa Swayne of the Confocal and Specialized Microscopy Core for her microscopy expertise. We particularly appreciate the assistance of Mark Graham, Richard Lee, and Rosanne Crooke of Ionis Pharmaceuticals, who provided the 3 ASOs used in this work as well as important advice. DC was supported by an NIH training grant (5T32DK007328-33). This work was supported by a grant from the National Heart, Lung, and Blood Institute (NHLBI) (R01 HL55638).

Address correspondence to: Henry N. Ginsberg, MD, Department of Medicine, PH 10-305, Columbia University, 630 West 168th Street, New York, New York 10032, USA. Phone: 212.305.9562; E-mail:hng1@columbia.edu.
1. Chapman MJ, et al. Triglyceride-rich lipoproteins and high-density lipoprotein cholesterol in patients at high risk of cardiovascular disease: evidence and guidance for management. Eur Heart J. 2011;32(11):1345-1361.

2. Choi SH, Ginsberg HN. Increased very low density lipoprotein (VLDL) secretion, hepatic steatosis, and insulin resistance. Trends Endocrinol Metab. 2011;22(9):353-363.

3. Tanoli T, Yue P, Yablonskiy D, Schonfeld G. Fatty liver in familial hypobetalipoproteinemia: roles of the APOB defects, intra-abdominal adipose tissue, and insulin sensitivity. J Lipid Res. 2004;45(5):941-947.

4. Young SG. Recent progress in understanding apolipoprotein B. Circulation. 1990;82(5):1574-1594.

5. Ginsberg HN, Fisher EA. The ever-expanding role of degradation in the regulation of apolipoprotein B metabolism. J Lipid Res. 2009; 50(Suppl):S162-S166.

6. Reyes-Soffer G, Ginsberg HN. Specail Patient Populations: Diabetes and Metabolic Syndrome. In: Clinical Lipidology, Companion to Braunwald's Heart Disease. Philadelphia, PA: Elsevier; 2014:401-417.

7. Raal FJ, et al. Mipomersen, an apolipoprotein B synthesis inhibitor, for lowering of LDL cholesterol concentrations in patients with homozygous familial hypercholesterolaemia: a randomised, double-blind, placebo-controlled trial. Lancet. 2010;375(9719):998-1006.
8. Cuchel M, et al. Efficacy and safety of a microsomal triglyceride transfer protein inhibitor in patients with homozygous familial hypercholesterolaemia: a single-arm, open-label, phase 3 study. Lancet. 2013;381(9860):40-46.

9. Crooke RM, Graham MJ, Lemonidis KM, Whipple CP, Koo S, Perera RJ. An apolipoprotein B antisense oligonucleotide lowers LDL cholesterol in hyperlipidemic mice without causing hepatic steatosis. J Lipid Res. 2005;46(5):872-884.

10. Lee RG, et al. Comparison of the pharmacological profiles of murine antisense oligonucleotides targeting apolipoprotein B and microsomal triglyceride transfer protein. J Lipid Res. 2013;54(3):602-614.

11. Cuchel M, Rader DJ. Is the cholesteryl ester transfer protein proatherogenic or antiatherogenic in humans?. J Am Coll Cardiol. 2007;50(20):1956-1958.

12. Wierzbicki AS, Hardman T, Prince WT. Future challenges for microsomal transport protein inhibitors. Curr Vasc Pharmacol. 2009;7(3):277-286.

13. Reid BN, et al. Hepatic overexpression of hormone-sensitive lipase and adipose triglyceride lipase promotes fatty acid oxidation, stimulates direct release of free fatty acids, and ameliorates steatosis. J Biol Chem. 2008;283(19):13087-13099.

14. Mizushima N, Yoshimori T. How to interpret LC3 immunoblotting. Autophagy. 2007;3(6):542-545.

15. Singh R, et al. Autophagy regulates lipid metabolism. Nature. 2009;458(7242):1131-1135.
16. Korolchuk VI, et al. Lysosomal positioning coordinates cellular nutrient responses. Nat Cell Biol. 2011;13(4):453-460.

17. Pankiv S, et al. p62/SQSTM1 binds directly to Atg8/LC3 to facilitate degradation of ubiquitinated protein aggregates by autophagy. J Biol Chem. 2007;282(33):24131-24145.

18. Klionsky DJ, et al. Guidelines for the use and interpretation of assays for monitoring autophagy (3rd edition). Autophagy. 2016;12(1):1-222.

19. Lippai M, Lőw P. The role of the selective adaptor p62 and ubiquitin-like proteins in autophagy. Biomed Res Int. 2014;2014:832704.

20. Ota T, Gayet C, Ginsberg HN. Inhibition of apolipoprotein B100 secretion by lipid-induced hepatic endoplasmic reticulum stress in rodents. J Clin Invest. 2008;118(1):316-332.

21. Fu S, Watkins SM, Hotamisligil GS. The role of endoplasmic reticulum in hepatic lipid homeostasis and stress signaling. Cell Metab. 2012;15(5):623-634.

22. Bernales S, McDonald KL, Walter P. Autophagy counterbalances endoplasmic reticulum expansion during the unfolded protein response. PLoS Biol. 2006;4(12):e423.

23. Ktistakis NT, Karanasios E, Manifava M. Dynamics of autophagosome formation: a pulse and a sequence of waves. Biochem Soc Trans. 2014;42(5):1389-1395.

24. Bains M, Florez-McClure ML, Heidenreich KA. Insulin-like growth factor-I prevents the accu- 
mulation of autophagic vesicles and cell death in Purkinje neurons by increasing the rate of autophagosome-to-lysosome fusion and degradation. J Biol Chem. 2009;284(30):20398-20407.

25. Dobbins RL, Szczepaniak LS, Bentley B, Esser V, Myhill J, McGarry JD. Prolonged inhibition of muscle carnitine palmitoyltransferase-1 promotes intramyocellular lipid accumulation and insulin resistance in rats. Diabetes. 2001;50(1):123-130.

26. Sridhar S, et al. The lipid kinase PI4KIII $\beta$ preserves lysosomal identity. ЕMBO J. 2013;32(3):324-339.

27. Fu S, et al. Aberrant lipid metabolism disrupts calcium homeostasis causing liver endoplasmic reticulum stress in obesity. Nature. 2011;473(7348):528-531.

28. Ravikumar B, et al. Regulation of mammalian autophagy in physiology and pathophysiology. Physiol Rev. 2010;90(4):1383-1435.

29. Anderson N, Borlak J. Molecular mechanisms and therapeutic targets in steatosis and steatohepatitis. Pharmacol Rev. 2008;60(3):311-357.

30. Wree A, Broderick L, Canbay A, Hoffman HM, Feldstein AE. From NAFLD to NASH to cirrhosisnew insights into disease mechanisms. Nat Rev Gastroenterol Hepatol. 2013;10(11):627-636.

31. Singh R. Autophagy and regulation of lipid metabolism. Results Probl Cell Differ. 2010;52:35-46.

32. Deegan S, Saveljeva S, Gorman AM, Samali A. Stress-induced self-cannibalism: on the regulation of autophagy by endoplasmic reticulum stress. Cell Mol Life Sci. 2013;70(14):2425-2441.

33. Wilfling F, Haas JT, Walther TC, Farese RV. Lipid droplet biogenesis. Curr Opin Cell Biol.
2014;29:39-45.

34. Finn PF, Dice JF. Proteolytic and lipolytic responses to starvation. Nutrition. 2006;22(7-8):830-844.

35. Singh R, Cuervo AM. Lipophagy: connecting autophagy and lipid metabolism. Int J Cell Biol. 2012;2012:282041.

36. Hussain MM, Shi J, Dreizen P. Microsomal triglyceride transfer protein and its role in apoB-lipoprotein assembly. J Lipid Res. 2003;44(1):22-32.

37. Schröder M, Kaufman RJ. ER stress and the unfolded protein response. Mutat Res. 2005;569(1-2):29-63.

38. Wang S, Kaufman RJ. How does protein misfolding in the endoplasmic reticulum affect lipid metabolism in the liver? Curr Opin Lipidol. 2014;25(2):125-132.

39. Alexander CA, Hamilton RL, Havel RJ. Subcellular localization of $\mathrm{B}$ apoprotein of plasma lipoproteins in rat liver. JCell Biol. 1976;69(2):241-263.

40. Hamilton RL, Wong JS, Cham CM, Nielsen LB, Young SG. Chylomicron-sized lipid particles are formed in the setting of apolipoprotein B deficiency. J Lipid Res. 1998;39(8):1543-1557.

41. Schuck S, Prinz WA, Thorn KS, Voss C, Walter P. Membrane expansion alleviates endoplasmic reticulum stress independently of the unfolded protein response. JCell Biol. 2009;187(4):525-536.

42. Zhang S, et al. Inhibition of autophagy promotes caspase-mediated apoptosis by tunicamycin in HepG2 cells. Toxicol Mech Methods. 2014;24(9):654-665.

43. Qin L, Wang Z, Tao L, Wang Y. ER stress negatively regulates AKT/TSC/mTOR pathway to enhance autophagy. Autophagy. 2010;6(2):239-247.
44. Chen X, Yin XM. Coordination of autophagy and the proteasome in resolving endoplasmic reticulum stress. Vet Pathol. 2011;48(1):245-253.

45. Wang $\mathrm{H}$, et al. Restoration of autophagy alleviates hepatic ER stress and impaired insulin signalling transduction in high fructose-fed male mice. Endocrinology. 2015;156(1):169-181.

46. Yorimitsu T, Klionsky DJ. Endoplasmic reticulum stress: a new pathway to induce autophagy. Autophagy. 2007;3(2):160-162.

47. Bernales S, Schuck S, Walter P. ER-phagy: selective autophagy of the endoplasmic reticulum. Autophagy. 2007;3(3):285-287.

48. Lipatova Z, Segev N. A role for macro-ERphagy in ER quality control. PLoS Genet. 2015;11(7):e1005390.

49. Schuck S, Gallagher CM, Walter P. ER-phagy mediates selective degradation of endoplasmic reticulum independently of the core autophagy machinery. J Cell Sci. 2014;127(Pt 18):4078-4088.

50. Mochida K, et al. Receptor-mediated selective autophagy degrades the endoplasmic reticulum and the nucleus. Nature. 2015;522(7556):359-362.

51. Khaminets A, et al. Regulation of endoplasmic reticulum turnover by selective autophagy. Nature. 2015;522(7556):354-358.

52. Gibbons GF, Wiggins D, Brown AM, Hebbachi AM. Synthesis and function of hepatic verylow-density lipoprotein. Biochem Soc Trans. 2004;32(Pt 1):59-64.

53. Lehner R, Lian J, Quiroga AD. Lumenal lipid metabolism: implications for lipoprotein assembly. Arterioscler Thromb Vasc Biol. 2012;32(5):1087-1093. 